\title{
GLOBAL SOLUTION TO A NON-LINEAR WAVE EQUATION OF LIQUID CRYSTAL IN THE CONSTANT ELECTRIC FIELD
}

\author{
LINJUN HUANG
}

\begin{abstract}
We construct a global conservative weak solution to the Cauchy problem for the non-linear variational wave equation $v_{t t}-c(v)\left(c(v) v_{x}\right)_{x}+\frac{1}{2} g(v)=0$ where $g(v)$ is defined in $(2.5)$ and $c(\cdot)$ is any smooth function with uniformly positive bounded value. This wave equation is derived from a wave system modelling nematic liquid crystals in a constant electric field.
\end{abstract}

\section{INTRODUCTION}

\subsection{Physical background.}

In this paper, we study a wave equation modelling the nematic liquid crystal in one space dimension with electric field applied. In the nematic phase, the orientation of the molecules can be described by a field of unit vector $\mathbf{n}(x, t) \in S^{2}$, the unit sphere. The famous OseenFrank potential energy density $W$ associated with the director field $\mathbf{n}$ is defined by

$$
W(\mathbf{n}, \nabla \mathbf{n})=\alpha|\mathbf{n} \times(\nabla \times \mathbf{n})|^{2}+\beta(\nabla \cdot \mathbf{n})^{2}+\gamma(\mathbf{n} \cdot \nabla \times \mathbf{n})^{2},
$$

where $\alpha, \beta$ and $\gamma$ are positive elastic constants of the liquid crystal. $\alpha$ represents the splay phenomenon of the nematic liquid crystal, $\beta$ represents the bend phenomenon, and $\gamma$ represents the twist phenomenon. When the kinetic energy are neglected in studies of nematic liquid crystals, by variational principle, we obtain an elliptic partial differential equation [11]. When we include the kinetic energy on modelling the nematic liquid crystal in one space dimension without any fields applied, we can formulate it as a non-linear wave equation which is derived in [7]:

$$
u_{t t}-c(u)\left[c(u) u_{x}\right]_{x}=0
$$

with smooth function $u$.

We study the nematic liquid crystal under the a constant electric field with the electric energy density described by

$$
f_{\text {electric }}=-\frac{1}{2} \mathbf{P} \cdot \mathbf{E}=\frac{1}{2} \varphi \mathbf{E}^{2}+\frac{1}{2} \eta(\mathbf{E} \cdot \mathbf{n})^{2},
$$

where $\mathbf{P}$ is the polarization, $\mathbf{E}$ is the electric field. We assume that the applied field is neither parallel nor perpendicular to $\mathbf{n} . \varphi$ and $\eta$ are positive constants related to permittivity and dielectric constants [12].

\subsection{Known results.}

For the equation (1.1), Glassey, Hunter, and Zheng [7] showed that the smooth solutions 
develop singularities in finite time. Also, Zhang and Zheng [19] studied that under weak conditions on the initial data which allow the solutions to have blow-up singularities and they established approximate solutions with estimates along precompactness using Young measure methods.

Our main reference is [5]. For the Cauchy problem for (1.1) with initial data $u(0, x)=$ $u_{0}(x), u_{t}(0, x)=u_{1}(x)$, Bressan and Zheng [5] proved the existence of a conservative weak solution by method of characteristics. They constructed conservative weak solution by introducing new sets of dependent and independent variables and showed that the solution can be obtained as the fixed point of a contraction transformation. See also [8]. Compared with [5], our energy equation has new terms from the applied electric field. These terms can be expressed as $G(v)$ where $G(v)$ is defined in (2.6). To solve this problem, we need to do some modification on the proof in [5] based on the observation that $v \in H^{1}$ and $G(v)$ is the lower order term in the energy equation.

For the Cauchy problem for (1.1) with initial data, Bressan, Chen and Zhang [3] proved the uniqueness of conservative solutions. Brassan and Huang [4] constructed dissipative solutions for $c^{\prime}>0$ relying on Kolmogorov's compactness theorem. Zhang and Zheng studied the existence and regularity properties of classical and weak solutions using the Young measure theory in [18] and proved the global existence of weak solutions in [20]. For $C^{3}$ initial data, Bressan and Chen [1] showed that the conservative solutions are piecewise smooth in $t-x$ plane. In [2], Bressan and Chen constructed a metric that renders the flow uniformly Lipschitz continuous on bounded subsets of $H^{1}(\mathbb{R})$. Zhang and Zheng [21] studied the existence of global weak solutions to the initial value problem (1.1) with general initial data $\left(u(0), u_{t}(0)\right)=\left(u_{0}, u_{1}\right) \in W^{1,2} \times L^{2}$ with wave speed satisfying $c^{\prime}(\cdot) \geq 0$ and $c^{\prime}\left(u_{0}(\cdot)\right)>0$.

For a wave system modelling nematic liquid crystals in one space dimension, Chen and Zheng [6] studied the global existence and singularity formation. Huang and Zheng [9] established the global existence of smooth solutions. Zhang and Zheng [13] constructed a weak global solutions to the Cauchy problem for a system of two variational wave equations on the real line and [14] showed the global weak solutions to the initial value problem for a complete system of variational wave equations modelling liquid crystals in one space dimension.

[10] shows that the weakly nonlinear unidirectional waves satisfying (1.1) are discribed asymptotically by

$$
\left(u_{t}+u^{n} u_{x}\right)_{x}=\frac{1}{2} n u^{n-1}\left(u_{x}\right)^{2}
$$

derived by Hunter and Saxton via weakly nonlinear geometric optics. In [15]-[17], Zhang and Zheng studied the global existence, uniqueness, and regularity of the dissipative and conservative solutions to $(1.2)(n=1,2)$ with $L^{2}$ initial data.

\subsection{Main theorems.}

Our main results are stated as follows. For the nematic liquid crystal under electric field, 
we obtain a Cauchy problem

$$
v_{t t}-c(v)\left(c(v) v_{x}\right)_{x}+\frac{1}{2} g(v)=0
$$

with the initial data

$$
v(0, x)=v_{0}(x), \quad v_{t}(0, x)=v_{1}(x),
$$

and $g(v)$ is defined in (2.5).

For the smooth function $c(\cdot)$, we assume that $c: \mathbb{R} \mapsto \mathbb{R}^{+}$is a bounded and uniformly positive function.

Definition 1.1. The definition of weak solution.

We say that for all test function $\phi \in C_{c}^{1}$, the function $v \in H^{1}$ satisfies the following integral:

$$
\iint \phi_{t} v_{t}-[c(v) \phi]_{x}\left[c(v) v_{x}\right]-\frac{\phi}{2} g(v) d x d t=0,
$$

is a weak solution to the equation (1.3).

Definition 1.2. The definition of energy conservative weak solution.

For $v_{1}$ and $v_{0}$ defined in (1.4) and $G(v)$ is defined in (2.6), we define the ground state energy $\mathcal{E}_{0}$ as:

$$
\mathcal{E}_{0}:=\frac{1}{2} \int\left\{v_{1}^{2}(x)+c^{2}\left(v_{0}(x)\right)\left[v_{0}^{2}(x)\right]_{x}+G\left(v_{0}(x)\right)\right\} d x .
$$

The function $v \in H^{1}$ is a energy conservative weak solution if it satisfying

$$
\mathcal{E}(t):=\frac{1}{2} \int\left\{v_{t}^{2}(t, x)+c^{2}(v(t, x)) v_{x}^{2}(t, x)+G(v(t, x))\right\} d x=\mathcal{E}_{0},
$$

for almost every $t \in \mathbb{R}$.

Theorem 1.1. Assume that $c: \mathbb{R} \mapsto\left[\mathcal{K}^{-1}, \mathcal{K}\right]$ is a smooth function for some $\mathcal{K}>1$. $v_{0}(x)$ and $v_{1}(x)$ are stated in (1.4). Also assume that the initial data $v_{0}(x)$ is absolutely continuous, $\left(v_{0}(x)\right)_{x} \in H^{1}$, and $v_{1}(x) \in H^{1}$. Then (1.3)-(1.4) can be considered as a Cauchy problem admitting a weak solution $v(t, x)$ defined for all $(t, x) \in \mathbb{R} \times \mathbb{R}$. Moreover, in the $t$ - $x$ plane, $v(x, t)$ is locally Hölder- $\frac{1}{2}$ continuous. For all $1 \leq p<2$, the map $t \mapsto v(t, \cdot)$ is continuously differentiable with values in $L_{l o c}^{p}$. The weak solution $v(t . \cdot)$ is Lipschitz continuous with respect to $L^{2}$ distance. So, for all $t, s \in \mathbb{R}$,

$$
\|v(t, \cdot)-v(s, \cdot)\|_{L^{2}} \leq L|t-s| .
$$

For all test function $\phi \in C_{c}^{1}$, the equation (1.3) satisfies (1.5).

Theorem 1.2. A family of weak solutions to the Cauchy problem (1.3)-(1.4) can be obtained with the properties:

$$
\mathcal{E}(t) \leq \mathcal{E}_{0}
$$


Let a sequence of initial condition satisfies:

$$
\begin{gathered}
\left\|\left(v_{0}^{n}(x)\right)_{x}-\left(v_{0}(x)\right)_{x}\right\|_{L^{2}} \rightarrow 0, \\
\left\|v_{1}^{n}(x)-v_{1}(x)\right\|_{L^{2}} \rightarrow 0 .
\end{gathered}
$$

Also, $u^{n} \rightarrow u$ uniformly on bounded subsets of the $t-x$ plane and $v_{0}^{n} \rightarrow v_{0}$ on compact sets as $n \rightarrow \infty$.

Theorem 1.3. There exists a continuous family of positive Radon measures $\left\{\mu_{t}: t \in \mathbb{R}\right\}$. This family of positive Radon measure is defined on the real line and it satisfies the following properties:

(i) $\mu_{t}(\mathbb{R})=\mathcal{E}_{0}$ for any time $t$.

(ii) With respect to Lebesgue measure, the absolutely continuous part of $\mu_{t}$ has density $\frac{1}{2}\left(v_{t}^{2}+\right.$ $\left.c^{2}(v) v_{x}^{2}+G(v)\right)$.

(iii) The singular part of $\mu_{t}$ has measure zero on the set where $c^{\prime}(v)=0$.

The paper is organized as follows. In section 2, we derive the energy equation and introduce a new set of dependent variables. Based on those dependent variables, we formulate a set of equations in terms of the new variables. This set of equations is equivalent to (1.3). In section 3, we use a transformation in a Banach space. In the transformation, we find the suitable weighted norm. This shows that there is a unique solution to the set of equations in terms of the new variables. In section 4, we show that the integral (1.5) holds and the Hölder- $\frac{1}{2}$ continuous condition holds. In section 5 , we show that (1.9) holds and the Lipschitz condition on the map $t \mapsto v(t, \cdot)$ and provide a proof of Theorem 1.2. On section 6 , we study the maps of $t \mapsto u_{x}(t, \cdot)$ and $t \mapsto u_{t}(t, \cdot)$, and complete the proof of Theorem 1.1. We provide a proof of Theorem 1.3 in section 7 .

\section{VARIABle Transformations}

2.1. Derivation of (1.3). Equation (1.3) has some physical origins. In the context of nematic liquid crystals, we introduce the famous Oseen-Frank potential energy density $W$ is given by

$$
W(\mathbf{n}, \nabla \mathbf{n})=\alpha|\mathbf{n} \times(\nabla \times \mathbf{n})|^{2}+\beta(\nabla \cdot \mathbf{n})^{2}+\gamma(\mathbf{n} \cdot \nabla \times \mathbf{n})^{2} .
$$

As stated in [12], in a electric field, the electric energy of the liquid crystal per unit volume is given by

$$
f_{\text {electric }}=-\frac{1}{2} \mathbf{P} \cdot \mathbf{E}=\frac{1}{2} \varphi \mathbf{E}^{2}+\frac{1}{2} \eta(\mathbf{E} \cdot \mathbf{n})^{2} .
$$

We discuss that when the electric energy is low when the applied electric field is normal to the liquid crystal director. And $\varphi$ and $\eta$ are some positive constants related to the permittivity so that (2.2) is equivalent to $\left|\mathbf{n} \cdot \mathbf{E}^{\perp}\right|^{2}+1$. And we denote $\mathbf{E}^{\perp}$ as a vector such that $\mathbf{E} \cdot \mathbf{E}^{\perp}=0$ and $\mathbf{E}^{\perp}=(1,0)$. So, the electric energy can be described as $\left|\mathbf{n} \cdot \mathbf{E}^{\perp}\right|^{2}$. By 
the property of the potential energy, the action can be describe as

$$
S=\iint\left|\mathbf{n}_{t}\right|^{2}-W(\mathbf{n}, \nabla \mathbf{n})-\left|\mathbf{n} \cdot \mathbf{E}^{\perp}\right|^{2} d x d t
$$

By plug in $\mathbf{n}=(\cos u, \sin u)$, the action can be describe as:

$$
S=\iint u_{t}^{2}-(c(u))^{2} u_{x}^{2}-(\cos u)^{2} d x d t
$$

We let $v=u-\frac{\pi}{2}$ so that $\cos u=\sin v$. By the principle of least action,

$$
\delta S=0=\iint \delta\left[v_{t}^{2}-(c(v))^{2} v_{x}^{2}-(\sin v)^{2}\right] d x d t .
$$

A straightforward computation shows that

$$
v_{t t}-c(v)\left(c(v) v_{x}\right)_{x}+\frac{g(v)}{2}=0
$$

where

$$
g(v)=2 \sin v \cos v .
$$

And define $G(v)$ as two times the anti derivative of $g(v)$,

$$
G(v)=2(\sin v)^{2} .
$$

\subsection{Derivation of the energy equation.}

From (2.4), we can compute that

$$
\begin{aligned}
& \int v_{t} v_{t t}-v_{t} c(v)\left[c(v) v_{x}\right]_{x}+\frac{g(v)}{2} v_{t} d x=0 \\
& \int\left(\frac{1}{2} v_{t}^{2}\right)_{t}+\left(\frac{c^{2}(v) v_{x}^{2}}{2}\right)_{t}+\left(\frac{1}{2} G(v)\right)_{t} d x=0 .
\end{aligned}
$$

And from (2.7), the energy equation can be described as

$$
E:=\frac{1}{2}\left(v_{t}^{2}+c^{2}(v) v_{x}^{2}+G(v)\right) .
$$

2.3. Variables transform. In this section we derive identities that holds for smooth solutions. The variable transformations are inspired by Bressan-Zheng [5]. We first denote variables:

$$
\left\{\begin{array}{l}
R:=v_{t}+c(v) v_{x}, \\
S:=v_{t}-c(v) v_{x} .
\end{array}\right.
$$

Thus, we can write $v_{t}$ and $v_{x}$ as follows

$$
\left\{\begin{array}{l}
v_{t}=\frac{R+S}{2}, \\
v_{x}=\frac{R-S}{2 c} .
\end{array}\right.
$$


By (1.3), the following identities are valid :

$$
\left\{\begin{array}{l}
S_{t}+c S_{x}=\frac{c^{\prime}}{4 c}\left(S^{2}-R^{2}\right)-\frac{1}{2} g(v), \\
R_{t}-c R_{x}=\frac{c^{\prime}}{4 c}\left(R^{2}-S^{2}\right)-\frac{1}{2} g(v),
\end{array}\right.
$$

by the following calculation

$$
\begin{aligned}
R_{t}-c R_{x} & =\left(v_{t}+c v_{x}\right)_{t}-c\left(v_{t}+c v_{x}\right)_{x} \\
& =g(v)+\frac{c^{\prime}}{4 c}\left(R^{2}-S^{2}\right) .
\end{aligned}
$$

We can compute $S_{t}+c S_{x}$ in the similar way to get (2.11) and denote energy and momentum as

$$
\begin{gathered}
E:=\frac{1}{2}\left(v_{t}^{2}+c^{2}(v) v_{x}^{2}+G(v)\right)=\frac{R^{2}+S^{2}}{4}+\frac{G(v)}{2}, \\
M:=-v_{t} v_{x}=\frac{S^{2}-R^{2}}{4 c} .
\end{gathered}
$$

The analysis of (1.3) has a main difficult that the possible breakdown of the regularity solutions. The quantities $v_{x}$ and $v_{t}$ can blow up in finite time even with smooth initial data. Thus we need to introduce a new set of dependent variables to deal with the possible unbounded value $R$ and $S$ :

$$
w:=2 \arctan R, \quad z:=2 \arctan S .
$$

Thus

$$
R=\tan \left(\frac{w}{2}\right), \quad S=\tan \left(\frac{z}{2}\right)
$$

By (2.11),

$$
\begin{aligned}
w_{t}-c w_{x} & =\frac{2}{1+R^{2}}\left(R_{t}-c R_{x}\right)=\frac{c^{\prime}}{2 c} \frac{R^{2}-S^{2}}{1+R^{2}}-\frac{g(v)}{1+R^{2}}, \\
z_{t}+c z_{x} & =\frac{2}{1+S^{2}}\left(S_{t}+c S_{x}\right)=\frac{c^{\prime}}{2 c} \frac{S^{2}-R^{2}}{1+S^{2}}-\frac{g(v)}{1+S^{2}} .
\end{aligned}
$$

In order to reduce the equation to a semi-linear system, we need to have a further change of variables. The forward characteristics equation and the backward characteristics equation:

$$
\dot{x}^{+}=c(v), \quad \dot{x}^{-}=c(v) .
$$

And we denote the characteristics lines pass through the point $(t, x)$ as

$$
s \rightarrow x^{+}(s, t, x), \quad s \rightarrow x^{-}(s, t, x) .
$$

So we can use a new coordinate system $(X, Y)$ to represent point $(t, x)$ by

$$
\begin{aligned}
X & :=\int_{0}^{x^{-}(0, t, x)}\left(1+R^{2}(0, x)\right) d x, \\
Y & :=\int_{x^{+}(0, t, x)}^{0}\left(1+S^{2}(0, x)\right) d x .
\end{aligned}
$$




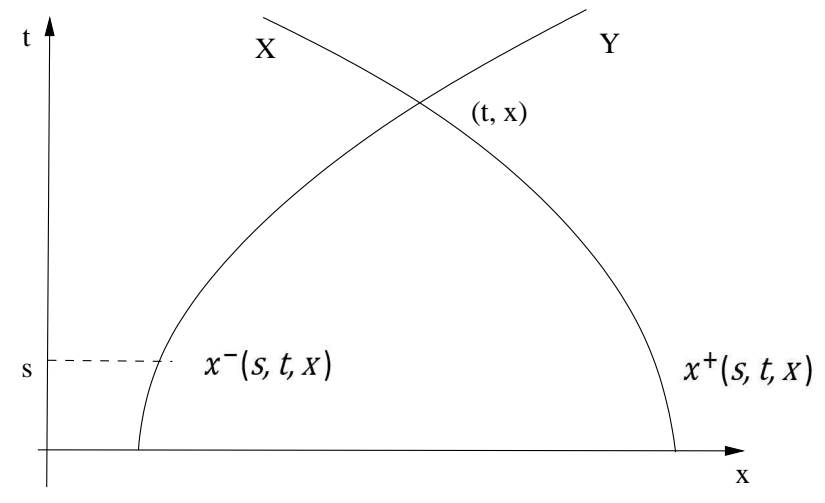

FIGURE 1. The characteristic curves.

(2.18) and (2.19) implies that

$$
\begin{aligned}
X_{t}-c(v) X_{x}=0, & Y_{t}+c(v) Y_{x}=0, \\
\left(X_{x}\right)_{t}-\left(c(v) X_{x}\right)_{x}=0, & \left(Y_{x}\right)_{t}+\left(c(v) Y_{x}\right)_{x}=0 .
\end{aligned}
$$

Thus, given any smooth function $f$, by using (2.20),

$$
\begin{aligned}
& f_{t}+c(v) f_{x}=2 c(v) X_{x} f_{X}, \\
& f_{t}-c(v) f_{x}=2 c(v) Y_{x} f_{Y} .
\end{aligned}
$$

From (2.20), $X_{t}+c(v) X_{x}=2 c(v) X_{x}$. To get (2.22) we compute directly

$$
\begin{gathered}
f_{t}+c(v) f_{x}=f_{X} X_{t}+f_{Y} Y_{t}+c(v) f_{X} X_{x}+c(v) f_{Y} Y_{x}=\left(X_{t}+c(v) X_{x}\right) f_{X}=2 c(v) X_{x} f_{X}, \\
f_{t}-c(v) f_{x}=f_{X} X_{t}+f_{Y} Y_{t}-c(v) f_{X} X_{x}-c(v) f_{Y} Y_{x}=\left(Y_{t}-c(v) Y_{x}\right) f_{Y}=2 c(v) Y_{x} f_{Y} .
\end{gathered}
$$

Introducing new variables

$$
p:=\frac{1+R^{2}}{X_{x}}, \quad q:=\frac{1+S^{2}}{-Y_{x}}
$$

From (2.23),

$$
\begin{aligned}
& \frac{1}{X_{x}}=\frac{p}{1+R^{2}}=p \cos ^{2}\left(\frac{w}{2}\right)=\frac{p(1+\cos w)}{2}, \\
& \frac{1}{-Y_{x}}=\frac{q}{1+S^{2}}=q \cos ^{2}\left(\frac{z}{2}\right)=\frac{q(1+\cos z)}{2} .
\end{aligned}
$$

By applying (2.16)-(2.17) to (2.17),

$$
\begin{aligned}
& w_{t}-c w_{x}=2 c \frac{1+S^{2}}{q} w_{Y}=\frac{c^{\prime}}{2 c} \frac{R^{2}-S^{2}}{1+R^{2}}+\frac{g(v)}{1+R^{2}}, \\
& z_{t}+c z_{x}=2 c \frac{1+R^{2}}{p} z_{x}=\frac{c^{\prime}}{2 c} \frac{S^{2}-R^{2}}{1+S^{2}}+\frac{g(v)}{1+S^{2}} .
\end{aligned}
$$

Thus, $w_{Y}$ and $z_{X}$ can be write as

$$
\begin{aligned}
& w_{Y}=\frac{c^{\prime}}{4 c^{2}} \frac{R^{2}-S^{2}}{1+R^{2}} \frac{q}{1+S^{2}}-\frac{q}{2 c} \frac{1}{1+S^{2}} \frac{g(v)}{1+R^{2}}, \\
& z_{X}=\frac{c^{\prime}}{4 c^{2}} \frac{S^{2}-R^{2}}{1+S^{2}} \frac{p}{1+R^{2}}-\frac{p}{2 c} \frac{1}{1+S^{2}} \frac{g(v)}{1+R^{2}} .
\end{aligned}
$$


So

$$
\left\{\begin{array}{l}
w_{Y}=\frac{c^{\prime}}{8 c^{2}}(\cos z-\cos w) q-\frac{q}{8 c} g(v)(1+\cos z)(1+\cos w), \\
z_{X}=\frac{c^{\prime}}{8 c^{2}}(\cos w-\cos z) p-\frac{p}{8 c} g(v)(1+\cos z)(1+\cos w) .
\end{array}\right.
$$

By using (2.21) and (2.24),

$$
\begin{aligned}
p_{t}-c p_{x} & =\frac{1}{X_{x}} 2 R\left(R_{t}-c R_{x}\right)-\frac{1}{X_{x}^{2}}\left[\left(X_{x}\right)_{t}-c\left(X_{x}\right)_{x}\right]\left(1+R^{2}\right) \\
& =\frac{c^{\prime}}{2 c} \frac{p}{1+R^{2}}\left[S\left(1+R^{2}\right)-R\left(1+S^{2}\right)\right]-\frac{p}{1+R^{2}} R g(v), \\
q_{t}+c q_{x} & =\frac{1}{-Y_{x}} 2 S\left(S_{t}-c S_{x}\right)-\frac{1}{-Y_{x}^{2}}\left[\left(-Y_{x}\right)_{t}+c\left(-Y_{x}\right)_{x}\right]\left(1+S^{2}\right) \\
& =\frac{c^{\prime}}{2 c} \frac{q}{1+S^{2}}\left[R\left(1+S^{2}\right)-S\left(1+R^{2}\right)\right]-\frac{q}{1+S^{2}} S g(v) .
\end{aligned}
$$

By applying (2.22),

$$
\begin{gathered}
p_{t}-c p_{x}=-2 c Y_{x} p_{Y}, \\
q_{t}+c q_{x}=2 c X_{x} q_{X} .
\end{gathered}
$$

And thus,

$$
\begin{aligned}
p_{Y} & =\left(p_{t}-c p_{x}\right) \frac{1}{-2 c Y_{x}}=\left(p_{t}-c p_{x}\right) \frac{1}{2 c} \frac{q}{1+S^{2}} \\
& =\frac{c^{\prime}}{8 c^{2}}[\sin z-\sin w] p q-\frac{1}{8 c} p q \sin w g(v)(1+\cos z), \\
q_{X} & =\left(q_{t}+c q_{x}\right) \frac{1}{2 c X_{x}}=\left(q_{t}+c q_{x}\right) \frac{1}{2 c} \frac{p}{1+R^{2}} \\
& =\frac{c^{\prime}}{8 c^{2}}[\sin w-\sin z] p q-\frac{1}{8 c} p q \sin z g(v)(1+\cos w) .
\end{aligned}
$$

So, the following identities hold:

$$
\left\{\begin{array}{l}
p_{Y}=\frac{c^{\prime}}{8 c^{2}}[\sin z-\sin w] p q-\frac{1}{8 c} p q \sin w g(v)(1+\cos z), \\
q_{X}=\frac{c^{\prime}}{8 c^{2}}[\sin w-\sin z] p q-\frac{1}{8 c} p q \sin z g(v)(1+\cos w) .
\end{array}\right.
$$

Also, we plug in $f=v$ into the equation (2.22) and get

$$
\left\{\begin{array}{l}
v_{X}=\left(v_{t}+c v_{x}\right) \frac{1}{2 c} \frac{p}{1+R^{2}}=\frac{1}{2 c}\left(\tan \frac{w}{2} \cos ^{2} \frac{w}{2}\right) p=p \frac{1}{4 c} \sin w, \\
v_{Y}=\left(v_{t}-c v_{x}\right) \frac{1}{2 c} \frac{q}{1+S^{2}}=\frac{1}{2 c}\left(\tan \frac{z}{2} \cos ^{2} \frac{z}{2}\right) q=q \frac{1}{4 c} \sin z .
\end{array}\right.
$$

Combining (2.25), (2.26), and (2.27), we obtain a semi-linear hyperbolic system from the non-linear equation (1.3). This system uses $\mathrm{X}, \mathrm{Y}$ as independent variables with smooth 
coefficients for the variables $v, w, z, p, q$

$$
\begin{aligned}
& \left\{\begin{array}{l}
w_{Y}=\frac{c^{\prime}}{8 c^{2}}(\cos z-\cos w) q-\frac{q}{8 c} g(v)(1+\cos z)(1+\cos w), \\
z_{X}=\frac{c^{\prime}}{8 c^{2}}(\cos w-\cos z) p-\frac{p}{8 c} g(v)(1+\cos z)(1+\cos w),
\end{array}\right. \\
& \left\{\begin{array}{l}
p_{Y}=\frac{c^{\prime}}{8 c^{2}}[\sin z-\sin w] p q-\frac{1}{8 c} p q \sin w g(v)(1+\cos z), \\
q_{X}=\frac{c^{\prime}}{8 c^{2}}[\sin w-\sin z] p q-\frac{1}{8 c} p q \sin z g(v)(1+\cos w),
\end{array}\right. \\
& \left\{\begin{array}{l}
v_{X}=\frac{p}{4 c} \sin w, \\
v_{Y}=\frac{q}{4 c} \sin z,
\end{array}\right.
\end{aligned}
$$

The system (2.28)-(2.30) should have non-characteristic boundary conditions related to (1.4). From (1.4), $v_{0}$ and $v_{1}$ determine the initial values of $R$ and $S$ at time $t=0$. We denote the curve $\gamma$ as the line in $(X, Y)$ plane at time $t=0$, say

$$
Y=\varphi(X), \quad X \in \mathbb{R} .
$$

And $Y=\varphi(X)$ if and only if for some $x \in \mathbb{R}$,

$$
X=\int_{0}^{x}\left(1+R^{2}(0, x)\right) d x, \quad Y=\int_{x}^{0}\left(1+S^{2}(0, x)\right) d x .
$$

By the assumptions of the Theorem 1.1, $v_{0} \in H^{1}, v_{1} \in H^{1}$. This implies that $R \in H^{1}$ and $S \in H^{1}$. Moreover, in this case, we let

$$
\mathcal{E}_{0}:=\frac{1}{4} \int\left[R^{2}(0, x)+S^{2}(0, x)\right] d x<\infty .
$$

Thus,

$$
X(x):=\int_{0}^{x}\left(1+R^{2}(0, y)\right) d y, \quad Y(x):=\int_{x}^{0}\left(1+S^{2}(0, y)\right) d y .
$$

are absolutely continuous and well defined functions. Further more, by observing (2.32), $X$ is increasing and $Y$ is decreasing. So, we conclude that the map $X \mapsto \varphi(X)$ is continuous and decreasing. And from (2.31),

$$
|X+\varphi(X)| \leq 4 \mathcal{E}_{0}
$$

Since $(t, x) \in[0, \infty) \times(-\infty, \infty)$, so our new independent variables $(X, Y) \in \Omega^{+}$, and the domain is defined as

$$
\Omega^{+}:=\{(X, Y): Y \geq \varphi(X)\}
$$

along the curve

$$
\gamma:=\{(X, Y): Y=\varphi(X)\}
$$


We can have the following boundary data $(\bar{w}, \bar{z}, \bar{p}, \bar{q}, \bar{v}) \in L^{\infty}$,

$$
\begin{gathered}
\left\{\begin{array}{c}
\bar{w}=2 \arctan (R(0, x)), \\
\bar{z}=2 \arctan (S(0, x)),
\end{array}\right. \\
\left\{\begin{array}{l}
\bar{p} \equiv 1, \\
\bar{q} \equiv 1, \\
\bar{v}=v_{0}(x) .
\end{array}\right.
\end{gathered}
$$

\section{Construct the integral SOlution}

We prove the global existence and uniqueness for the semi-linear system $(2.28)-(2.30)$ in this section.

Theorem 3.1. If the assumptions of Theorem 1.1 holds, then the semi-linear system (2.28) - (2.30) with the boundary conditions (2.33) - (2.35) has a unique solution for all $(X, Y) \in$ $\mathbb{R} \times \mathbb{R}$.

We construct the solution on the region $\Omega^{+}$which is the case that $Y \geq \varphi(X)$. The proof of the solution on the $\Omega^{-}$which is the case that $Y \leq \varphi(X)$ can be construct in the similar way. We show the Lipschitz condition for the system (2.28) - (2.30). To make sure the solution is defined in the region $\Omega^{+}$, we need to construct some priori bounds. So that we can show that $p, q$ are bounded. The Lipschitz condition can be derived as follows. From the energy conservation equations (1.7), (1.6) and the assumption that $v \in H^{1}$, we denote the following constants:

$$
\begin{aligned}
& C_{1}:=\sup _{v(x, t) \in \mathbb{R}}\left|\frac{c^{\prime}(v)}{4 c^{2}(v)}\right|<\infty, \\
& K_{1}:=\sup _{t \geq 0} \int G(v) d x<\infty, \\
& K_{0}:=\sup _{x, t}|g(v)|<\infty .
\end{aligned}
$$

The variable transformation from $(x, t)$ to $(X, Y)$ changes the independent variable of $v$ not the dependent variable. Thus the boundedness of $K_{1}$ and $K_{0}$ remains.

From (2.29),

$$
\begin{aligned}
q_{X}+p_{Y}= & \frac{1}{2}[G(v) q(1+\cos z)]_{X}+\frac{1}{2}[G(v) p(1+\cos w)]_{Y} \\
& -\frac{1}{2} G(v) \frac{c^{\prime}}{8 c}(\sin w-\sin z)(\cos z-\cos w)\left(\frac{1}{c}+1\right) .
\end{aligned}
$$

We construct a closed curve $\Sigma$ for every $(X, Y) \in \Omega^{+}$with the vertical line segment connect $(X, Y)$ with $(X, \varphi(X))$, the horizontal line segment connect $(X, Y)$ with $\left(\varphi^{-1}(Y), Y\right)$, and a part of the boundary $\gamma=Y=\varphi(X)$ connecting $(X, \varphi(X))$ with $\left(\varphi^{-1}(Y), Y\right)$. The closed 


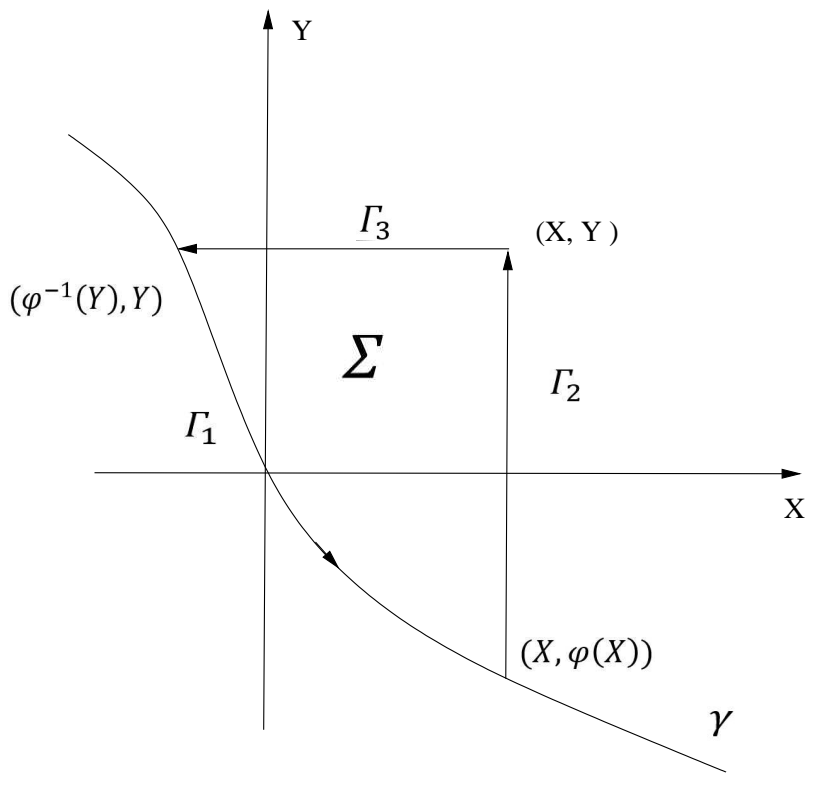

Figure 2. The closed curve $\Sigma$.

curve $\Sigma=\Gamma_{1}+\Gamma_{2}+\Gamma_{3}$. From (3.1), we compute $\iint q_{X}+p_{Y} d A=\int-p d X+\int q d Y$ and denote that

$$
\begin{aligned}
& \iint q_{X}+p_{Y} d A=\int-p d X+\int q d Y, \\
& Q_{X}:=\frac{1}{2}[G(v) q(1+\cos z)]_{X}, \\
& P_{Y}:=\frac{1}{2}[G(v) p(1+\cos w)]_{Y}, \\
& \xi:=G(v) \frac{c^{\prime}}{8 c}(\sin w-\sin z)(\cos z-\cos w)\left(\frac{1}{c}+1\right) .
\end{aligned}
$$

So

$$
\begin{aligned}
\iint_{\Omega} p_{y}+q_{X} d X d Y & =\int_{\Sigma}-p d X+\int_{\Sigma} q d Y \\
& =\iint_{\Omega} Q_{X}+P_{Y}-\frac{1}{2} \xi d X d Y,
\end{aligned}
$$

By Green's Theorem,

Thus,

$$
\int_{\Sigma}-p d X+\int_{\Sigma} q d Y=\int_{\Sigma}-P d X+\int_{\Sigma} Q d Y-\frac{1}{2} \iint_{\Omega} \xi d X d Y
$$

$$
\begin{aligned}
\frac{1}{2} \iint_{\Omega} \xi d X d Y & =\int_{\Sigma} p-P d X+\int_{\Sigma} Q-q d Y \\
& =\int_{\Sigma} p-\frac{G(v)}{2} p(1+\cos w) d X+\int_{\Sigma}-q+\frac{G(v)}{2} q(1+\cos z) d X
\end{aligned}
$$


Since $\Sigma=\Gamma_{1}+\Gamma_{2}+\Gamma_{3}$ is a closed curve, so we compute the integral of $\Gamma_{1}$ directly and in the way $\Gamma_{1}=-\left(\Gamma_{2}+\Gamma_{3}\right)$.

$$
\int_{\Gamma_{1}} 1-\frac{G(v)}{2}(1+\cos w) d X+\int_{\Gamma_{1}}-1+\frac{G(v)}{2}(1+\cos z) d Y
$$

and from $(2.24)$

$$
d X=\frac{2}{1+\cos w} d x, \quad d Y=\frac{2}{1+\cos z} d x .
$$

Thus $(1+\cos w) d X=2 d x$, and $(1+\cos w) d Y=2 d x$.

So (3.8) becomes

$$
\begin{aligned}
\int_{\Gamma_{1}} 1-\frac{G(v)}{2}(1+\cos w) d X+ & \int_{\Gamma_{1}}-1+\frac{G(v)}{2}(1+\cos z) d Y \\
& \leq 2\left(|X|+|Y|+4 \mathcal{E}_{0}\right)+K_{1} .
\end{aligned}
$$

And also,

$$
\begin{gathered}
\int_{\Gamma_{2}} 1-\frac{G(v)}{2}(1+\cos w) d X+\int_{\Gamma_{2}}-1+\frac{G(v)}{2}(1+\cos z) d Y \\
\leq 0-Y+\varphi(X)+\frac{K_{1}}{2}, \\
\int_{\Gamma_{3}} 1-\frac{G(v)}{2}(1+\cos w) d X+\int_{\Gamma_{3}}-1+\frac{G(v)}{2}(1+\cos z) d Y \\
\leq \varphi^{-1}(Y)-X-0+\frac{K_{1}}{2} .
\end{gathered}
$$

As a result,

$$
\int_{\varphi^{-1}(Y)}^{X} p\left(X^{\prime}, Y\right) d X^{\prime}+\int_{\varphi(X)}^{Y} q\left(X, Y^{\prime}\right) d Y^{\prime} \leq 2\left(|X|+|Y|+4 \mathcal{E}_{0}\right)+K_{1} .
$$

By observing the boundary conditions (2.33) - (2.35), $p, q>0$. And by (2.29),

$$
\begin{gathered}
p_{Y}=\frac{1}{8 c} p q\left\{\frac{c^{\prime}}{c}[\sin z-\sin w]-\sin w g(v)(1+\cos z)\right\}, \\
p(X, Y)=\exp \left\{\int_{\varphi(X)}^{Y} \frac{1}{8 c} \frac{c^{\prime}}{c}[\sin z-\sin w]-\sin w g(v)(1+\cos z) q\left(X, Y^{\prime}\right) d Y^{\prime}\right\} \\
\leq \exp \left\{C_{1} \int_{\varphi(X)}^{Y} q\left(X, Y^{\prime}\right) d Y^{\prime}\right\} \\
\leq \exp \left\{2 C_{1}\left(|X|+|Y|+4 \mathcal{E}_{0}\right)+C_{1} K_{1}\right\} .
\end{gathered}
$$

Similarly, we have

$$
q(X, Y) \leq \exp \left\{2 C_{1}\left(|X|+|Y|+4 \mathcal{E}_{0}\right)+C_{1} K_{1}\right\} .
$$

Now, we show that on any bounded sets in $X-Y$ plane, we can construct the solution for the system of the equations (2.28) - (2.30) with boundary condition (2.33) -(2.35) by the fixed point of a constructive map. For any $r>0$, we can construct a bounded domain

$$
\Omega_{r}:=\{(X, Y): Y \leq \varphi(X), X \leq r, Y \leq r\} .
$$


And also introduce the function space :

$$
\Lambda_{r}:=\left\{f: \Omega_{r} \mapsto \mathbb{R}:\|f\|_{*}:=\operatorname{esssup}_{(X, Y) \in \Omega_{r}} e^{-K(X+Y)}|f(X, Y)|<\infty\right\} .
$$

Where $K$ is a suitably big constant and it will be determined later. And for $(w, z, p, q, v) \in$ $\Lambda_{r}$, we construct a map $\tau(w, z, p, q, v)=(\tilde{w}, \tilde{z}, \tilde{p}, \tilde{q}, \tilde{v})$. And this map is define as follows.

$$
\begin{gathered}
\left\{\begin{array}{c}
\tilde{w}(X, Y)=\bar{w}(X, \varphi(X))+\int_{\varphi(X)}^{Y} \frac{c^{\prime}}{8 c^{2}}(\cos z-\cos w) q-\frac{q}{8 c} g(v)(1+\cos z)(1+\cos w) d Y, \\
\tilde{z}(X, Y)=\bar{z}\left(\varphi^{-1}(Y), Y\right)+\int_{\varphi^{-1}(Y)}^{X} \frac{c^{\prime}}{8 c^{2}}(\cos w-\cos z) p-\frac{p}{8 c} g(v)(1+\cos z)(1+\cos w) d X,
\end{array}\right. \\
(3.11) \quad\left\{\begin{array}{l}
\tilde{p}(X, Y)=1+\int_{\varphi(X)}^{Y} \frac{1}{8 c} p q\left\{\frac{c^{\prime}}{c}[\sin z-\sin w]-\sin w g(v)(1+\cos z)\right\} d Y, \\
\tilde{q}(X, Y)=1+\int_{\varphi^{-1}(Y)}^{X} \frac{1}{8 c} p q\left\{\frac{c^{\prime}}{c}[\sin w-\sin z]-\sin z(g(v)(1+\cos w)\} d X,\right. \\
\tilde{v}(X, Y)=\bar{v}(X, \varphi(X))+\int_{\varphi(X)}^{Y} \frac{1}{4 c} \sin z q d Y .
\end{array}\right.
\end{gathered}
$$

We want to prove the uniform Lipschitz condition by showing that $\Phi_{r}$ is a contracting map. First, we define

$$
\Phi_{r}:=\Lambda_{r} \times \Lambda_{r} \times \Lambda_{r} \times \Lambda_{r} \times \Lambda_{r} .
$$

For some properly chosen distance $D: \Phi_{r} \times \Phi_{r} \mapsto \mathbb{R}$, we want to show that

$$
D\left(\left(\tilde{w}_{1}, \tilde{z_{1}}, \tilde{p_{1}}, \tilde{q_{1}}, \tilde{v_{1}}\right),\left(\tilde{w}_{2}, \tilde{z_{2}}, \tilde{p_{2}}, \tilde{q_{2}}, \tilde{v_{2}}\right)\right)<L \times D\left(\left(w_{1}, z_{1}, p_{1}, q_{1}, v_{1}\right),\left(w_{2}, z_{2}, p_{2}, q_{2}, v_{2}\right)\right) .
$$

The Lipschitz constant $L$ satisfies $L \leq 1$. In fact, we define the distance as:

$$
\begin{aligned}
D\left(\left(\tilde{w_{1}}, \tilde{z_{1}}, \tilde{p_{1}}, \tilde{q_{1}}, \tilde{v_{1}}\right),\left(\tilde{w_{2}}, \tilde{z_{2}}, \tilde{p_{2}}, \tilde{q_{2}}, \tilde{v_{2}}\right)\right):=\max \left\{\left\|\tilde{w_{1}}-\tilde{w_{2}}\right\|_{*},\left\|\tilde{z_{1}}-\tilde{z_{2}}\right\|_{*},\right. \\
\left.\left\|\tilde{p_{1}}-\tilde{p_{2}}\right\|_{*},\left\|\tilde{q_{1}}-\tilde{q_{2}}\right\|_{*},\left\|\tilde{v_{1}}-\tilde{v_{2}}\right\|_{*}\right\},
\end{aligned}
$$

and the norm $\|\cdot\|_{*}$ is defined in (3.9).

A straightforward computation shows that $L=\frac{C\left(\mathcal{E}_{0}, \mathcal{K}\right)}{K}$, where $C\left(\mathcal{E}_{0}, \mathcal{K}\right)$ is a constant depends on $\mathcal{E}_{0}$ and $\mathcal{K}$. By choosing $K$ sufficiently large, we can guarantee $L<1$. Hence, the uniform Lipschitz condition is proved. By the fixed point theorem, the solution in the $X-Y$ plane exists and is unique.

If the initial data in (1.2) are smooth, then the solutions of (2.28) - (2.30) with boundary condition (2.33) - (2.35) are smooth functions with variables $(X, Y)$. Also, if there is a sequence of smooth functions $\left(v_{0}^{m}(x), v_{1}^{m}(x)\right)_{m \geq 1}$ with the following conditions:

$$
v_{0}^{m}(x) \rightarrow v_{0}(x), v_{1}^{m}(x) \rightarrow v_{1}(x),\left(v_{0}^{m}(x)\right)_{x} \rightarrow\left(v_{0}(x)\right)_{x},
$$

uniformly on a compact subset of $\mathbb{R}$. Then

$$
\left(p^{m}, q^{m}, w^{m}, z^{m}, v^{m}\right) \rightarrow(p, q, w, z, v),
$$

uniformly on some bounded subsets of $X-Y$ plane. 


\section{WEAK SOLUTIONS}

In this section, we construct a map $v(X, Y) \rightarrow v(t, x)$. That is to write $(X, Y)$ in terms of $(t, x)$ so we obtain a solution to the Cauchy problem (1.3), (1.4). The map $(X, Y) \mapsto(t, x)$ can be obtain in the following way. We plug in $f=x$ and $f=t$ into the equation (2.22), and get

$$
\left\{\begin{array}{l}
c=2 c X_{x} x_{X}, \\
-c=-2 c Y_{x} x_{Y}, \\
1=2 c X_{x} t_{X}, \\
1=-2 c Y_{x} t_{Y} .
\end{array}\right.
$$

And by applying (2.24) we have

$$
\left\{\begin{aligned}
X_{x} & =\frac{2}{(1+\cos w) p} \\
Y_{x} & =\frac{-2}{(1+\cos z) q} \\
X_{t} & =\frac{2 c}{(1+\cos w) p} \\
Y_{t} & =\frac{-2 c}{(1+\cos z) q}
\end{aligned}\right.
$$

We assume that the partial derivatives above valid for points that $w, z \neq-\pi$. Thus, we have

$$
\begin{aligned}
& \left\{\begin{array}{l}
x_{X}=\frac{1}{2 X_{x}}=\frac{(1+\cos w) p}{4}, \\
x_{Y}=\frac{1}{2 Y_{x}}=\frac{-(1+\cos z) q}{4},
\end{array}\right. \\
& \left\{\begin{array}{l}
t_{X}=\frac{1}{2 c X_{x}}=\frac{(1+\cos w) p}{4 c}, \\
t_{Y}=\frac{1}{-2 c Y_{x}}=\frac{(1+\cos z) q}{4 c} .
\end{array}\right.
\end{aligned}
$$

A computation shows that $x_{X Y}=x_{Y X}$ and $t_{X Y}=t_{Y X}$

$$
\begin{aligned}
x_{X Y} & =\frac{(1+\cos w) p_{Y}}{4}-\frac{p \sin w w_{Y}}{4} \\
& =\frac{c^{\prime} p q}{32 c}[\sin z-\sin w+\sin (z-w)], \\
x_{Y X} & =\frac{(1+\cos z) q_{X}}{4}-\frac{q \sin z z_{Y}}{4} \\
& =\frac{c^{\prime} p q}{32 c}[\sin z-\sin w+\sin (z-w)] .
\end{aligned}
$$

So, $x_{X Y}=x_{Y X}$.

And similarly, we can compute that $t_{X Y}=t_{Y X}$. Thus, the two equation in (4.3) are equivalent: $x_{X Y}=x_{Y X}$. And the two equation in (4.4) are equivalent since $t_{X Y}=t_{Y X}$. We 
can recover the solution in terms of $(t, x)$ with function $x=x(X, Y)$ by integrating one of the equation in (4.3). Also, we can write $t=t(X, Y)$ by integrating one of the equation in (4.4).

Next, we prove that the function $v$ is a weak solution to (1.3). From (1.5), we want to show that

$$
0=\iint \phi_{t} v_{t}-[c(v) \phi]_{x}\left[c(v) v_{x}\right]-\frac{\phi}{2} g(v) d x d t
$$

In fact, it is equivalent to prove:

$$
\begin{aligned}
0= & \iint\left(v_{t}+c v_{x}\right)\left[\phi_{t}-(c(v) \phi)_{x}\right]+\left(v_{t}-c v_{x}\right)\left[\phi_{t}+(c(v) \phi)_{x}\right]-\phi g(v) d x d t \\
= & \iint-\left(\frac{\sin w}{2} p\right)_{Y} \phi-\left(\frac{\sin z}{2} q\right)_{X} \phi+\frac{c^{\prime} p q}{8 c}\left[\sin w \frac{1+\cos z}{2}-\sin z \frac{1+\cos w}{2}\right] \phi\left(\tan \frac{z}{2}-\tan \frac{w}{2}\right) d X d Y \\
& -\iint \phi g(v) d x d t \\
:= & \text { I }+ \text { II. }
\end{aligned}
$$

We define I and II later, and where in the last step, we have used (4.2),

$$
d x d t=\left|\begin{array}{cc}
\frac{d x}{d X} & \frac{d x}{d Y} \\
\frac{d t}{d X} & \frac{d t}{d Y}
\end{array}\right| d X d Y=\frac{p q}{2 c\left(1+R^{2}\right)\left(1+S^{2}\right)} d X d Y .
$$

And used the following identities derived from (2.30),

$$
\begin{aligned}
& \left\{\begin{array}{l}
\frac{1}{1+R^{2}}=\frac{1+\cos w}{2}, \\
\frac{1}{1+S^{2}}=\frac{1+\cos z}{2},
\end{array}\right. \\
& \left\{\begin{array}{l}
\frac{R}{1+R^{2}}=\frac{\sin w}{2}, \\
\frac{S}{1+S^{2}}=\frac{\sin z}{2} .
\end{array}\right.
\end{aligned}
$$

We denote I and II as follows

$$
\begin{aligned}
\mathrm{I} & =\iint-\left(\frac{\sin w}{2} p\right)_{Y} \phi-\left(\frac{\sin z}{2} q\right)_{X} \phi \\
& +\frac{c^{\prime} p q}{8 c}\left[\sin w \frac{1+\cos z}{2}-\sin z \frac{1+\cos w}{2}\right] \phi\left(\tan \frac{z}{2}-\tan \frac{w}{2}\right) d X d Y,
\end{aligned}
$$

and

$$
\mathrm{II}=\iint \phi g(v) d x d t .
$$

A computation on I with (2.28) - (2.30) shows that

$$
\begin{aligned}
\mathrm{I} & =\iint-\left(\frac{\cos w}{2} w_{Y} p+\frac{\sin w}{2} p_{Y}\right) \phi-\left(\frac{\cos z}{2} z_{X} q+\frac{\sin z}{2} q_{X}\right) \phi+\frac{c^{\prime} p q}{8 c^{2}}[\cos (w+z)-1] \phi d X d Y \\
& =\iint \frac{p q}{16 c} \phi g(v)(\cos w+\cos z+2+2 \cos z \cos w
\end{aligned}
$$




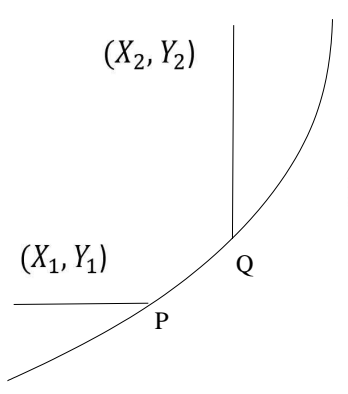

Case 1

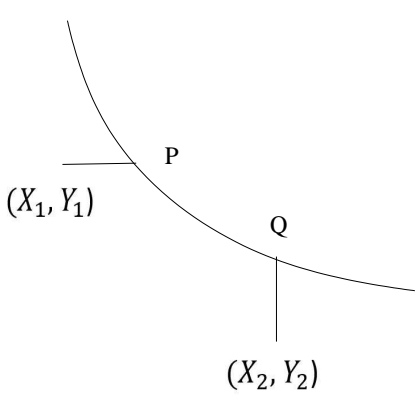

Case 2

FiguRE 3. The paths of integration.

$$
\begin{aligned}
& \left.+\cos ^{2} w \cos z+\cos w \cos ^{2} z+\sin ^{2} w \cos z+\sin ^{2} z \cos w\right) d X d Y \\
= & \iint \frac{p q}{8 c} \phi g(v)(1+\cos z+\cos w+\cos z \cos w) d X d Y .
\end{aligned}
$$

A computation on II shows that

$$
\begin{aligned}
\mathrm{II} & =\iint \phi g(v) \frac{p q}{2 c\left(1+s^{2}\right)\left(1+R^{2}\right)} d X d Y \\
& =\iint \frac{p q}{8 c} \phi g(v)(1+\cos z+\cos w+\cos z \cos w) d X d Y .
\end{aligned}
$$

Clearly, I = II. Thus the integral (1.5) holds since

$$
\begin{aligned}
0= & \iint-\left(\frac{\sin w}{2} p\right)_{Y} \phi-\left(\frac{\sin z}{2} q\right)_{X} \phi \\
& +\frac{c^{\prime} p q}{8 c}\left[\sin w \frac{1+\cos z}{2}-\sin z \frac{1+\cos w}{2}\right] \phi\left(\tan \frac{z}{2}-\tan \frac{w}{2}\right) d X d Y-\iint \phi g(v) d x d t \\
0= & \mathrm{I}-\mathrm{II},
\end{aligned}
$$

where I is defined in (4.7), and II is defined in (4.8).

Next, we define $v$ as a function in terms of the original variables $(t, x)$. We invert the map $(X, Y) \mapsto(t, x)$ and then we have $v(t, x)=v(X(t, x), Y(t, x))$. Given arbitrary $\left(t^{*}, x^{*}\right)$ in the $t$ - $x$ plane, we choose arbitrary point $\left(X^{*}, Y^{*}\right)$ in $X-Y$ plane such that $t^{*}=t\left(X^{*}, Y^{*}\right)$ and $x^{*}=x\left(X^{*}, Y^{*}\right)$. We define that $v\left(t^{*}, x^{*}\right)=v\left(X^{*}, Y^{*}\right)$ and assume that there are two different points $\left(t\left(X_{1}, Y_{1}\right), x\left(X_{1}, Y_{1}\right)\right)=\left(t\left(X_{2}, Y_{2}\right), x\left(X_{2}, Y_{2}\right)\right)=\left(t^{*}, x^{*}\right)$. We consider two cases: case 1: $X_{1} \leq X_{2}, Y_{1} \leq Y_{2}$, and case $2: X_{1} \leq X_{2}, Y_{1} \geq Y_{2}$. Case 1: $X_{1} \leq X_{2}, Y_{1} \leq Y_{2}$. We consider the set

$$
\Gamma_{x^{*}}:=\left\{(X, Y): x(X, Y) \leq x^{*}\right\} .
$$

We denote $\partial \Gamma_{x^{*}}$ as the boundary of $\Gamma_{x^{*}}$. By (4.3), we observe that $x$ is increasing with $X$ increasing and $x$ is decreasing with $Y$ increasing. Thus, this boundary can be write as a Lipschitz continuous function denoted as $X-Y=\phi(X-Y)$. We construct the Lipschitz continuous curve $\gamma$ with the following properties: 
- a horizontal line segment connecting $\left(X_{1}, Y_{1}\right)$ with a point $P=\left(X_{P}, Y_{P}\right) \in \partial \Gamma_{x^{*}}$ and $Y_{P}=Y_{1}$.

- a vertical line segment connecting $\left(X_{2}, Y_{2}\right)$ with a point $Q=\left(X_{Q}, Y_{Q}\right) \in \partial \Gamma_{x^{*}}$ and $X_{Q}=X_{2}$.

- a part of $\partial \Gamma_{x^{*}}$.

Thus, we obtain a Lipschitz continuous parametrization of the curve $\gamma:\left[\xi_{1}, \xi_{2}\right] \mapsto \mathbb{R} \times \mathbb{R}$ where the parameter $\xi=X+Y$. By observing, the map $(X, Y) \mapsto(t, x)$ is constant along the curve $\gamma$. And (4.3) - (4.4) implies that

$$
(1+\cos w) X_{\xi}=(1+\cos z) Y_{\xi}=0,
$$

From (4.9),

$$
\sin w X_{\xi}=\sin z Y_{\xi}=0 .
$$

Thus, by (4.10)

$$
\begin{aligned}
v\left(X_{2}, Y_{2}\right)-v\left(X_{1}, Y_{1}\right) & =\int_{\gamma}\left(v_{X} d X+v_{Y} d Y\right) \\
& =\int_{\xi_{1}}^{\xi_{2}}\left(\frac{p \sin w}{4 c} X_{\xi}-\frac{q \sin z}{4 c} Y_{\xi}\right) d \xi=0 .
\end{aligned}
$$

So our claim for case 1 is proved.

Case 2: $X_{1} \leq X_{2}, Y_{1} \geq Y_{2}$. We consider the set:

$$
\Gamma_{t^{*}}:=\left\{(X, Y): t(X, Y) \leq t^{*}\right\} .
$$

And we do the same process as we did in case 1 . Construct $\gamma$ connecting $\left(X_{1}, X_{2}\right)$ and $\left(X_{2}, Y_{2}\right)$ as Figure 3 case 2 indicates.

Next, we prove the function $v(t, x)=v(X(t, x), Y(t, x))$ is Hölder- $\frac{1}{2}$ continuous on the bounded sets. To prove this, we need to consider characteristic curve such that $t \mapsto x^{+}(t)$ with $\bar{x}^{+}=c(v)$. For some fixed $\bar{Y}$, this can be parametrized by the function $X \mapsto$ $(t(X, \bar{Y}), x(X, \bar{Y}))$. By $(2.20),(2.22),(2.24)$ and $(2.30)$,

$$
\begin{aligned}
\int_{0}^{\tau}\left[v_{t}+c(v) v_{x}\right]^{2} d t & =\int_{X_{0}}^{X_{\tau}}\left(2 c X_{x} v_{X}\right)^{2} \frac{1}{2 X_{t}} d X \\
& =\int_{X_{0}}^{X_{\tau}} \frac{p}{2 c} \sin ^{2}\left(\frac{w}{2}\right) d X \leq \int_{X_{0}}^{X_{\tau}} \frac{p}{2 c} d X \leq C_{\tau} .
\end{aligned}
$$

Thus, we obtain that

$$
\int_{0}^{\tau}\left[v_{t}+c(v) v_{x}\right]^{2} d t \leq C_{\tau} .
$$

Similarly, we integrate along backward characteristics curves $t \mapsto x^{-}(t)$ and find out that

$$
\int_{0}^{\tau}\left[v_{t}-c(v) v_{x}\right]^{2} d t \leq C_{\tau} .
$$


Thus, since the speed of the characteristic curve is $+c(v)$ or $-c(v)$ and $c(v)$ is uniformly positive bounded. With the bounds (4.11) and (4.12), the function $v(t, x)$ is Hölder- $\frac{1}{2}$ continuous.

\section{Conserved quANTIties}

This section provides a proof of Theorem 1.2. Recalling (2.12) and (2.13), a straightforward computation shows that

$$
\begin{aligned}
E_{t} & =\left(\frac{1}{2} v_{t}^{2}+\frac{1}{2} c^{2} v_{x}^{2}+\frac{G(v)}{2}\right)_{t} \\
& =v_{t t} v_{t}+c c^{\prime} v_{t} v_{x}^{2}+c^{2} v_{x} v_{x t}+g(v) v_{t}, \\
\left(c^{2} M\right)_{x} & =\left(-c^{2} v_{t} v_{x}\right)_{x}=-2 c c^{\prime} v_{x}^{2} v_{t}-c^{2} v_{t x} v_{x}-c^{2} v_{t} v_{x x}, \\
E_{t}+\left(c^{2} M\right)_{x} & =v_{t}\left(v_{t t}-c c^{\prime} v_{x}-c^{2} v_{x x}+\frac{1}{2} g(v)=0\right.
\end{aligned}
$$

and

$$
\begin{aligned}
M_{t} & =-v_{t t} v_{x}-v_{t} v_{x t}, \\
E_{x} & =v_{t} v_{t x}+c c^{\prime} v_{x} v_{x}^{2}+c^{2} v_{x} v_{x x}+\frac{1}{2} g(v) v_{x}, \\
M_{t}+E_{x} & =-v_{x}\left(v_{t t}-c c^{\prime} v_{x}^{2}-c^{2} v_{x x}-\frac{1}{2} g(v)\right)=0 .
\end{aligned}
$$

Thus,

$$
\left\{\begin{array}{l}
E_{t}+\left(c^{2} M\right)_{x}=0 \\
M_{t}+E_{x}=0
\end{array}\right.
$$

Also,

$$
\begin{aligned}
& d x=\frac{(1+\cos w) p}{4} d X-\frac{(1+\cos z) q}{4} d Y, \\
& d t=\frac{(1+\cos w) p}{4 c} d X+\frac{(1+\cos z) q}{4 c} d Y,
\end{aligned}
$$

which is closed. We want to show that $E d x-\left(c^{2} M\right) d t, M d x-E d t$ are closed. Recalling (2.28) - (2.30), we write $E d x-\left(c^{2} M\right) d t, M d x-E d t$ in terms of $X, Y$, and show that they are closed.

$$
\begin{aligned}
& E d x-\left(c^{2} M\right) d t= \\
& \quad\left[\frac{(1-\cos w)}{8}+\frac{(1+\cos w)}{8} G(v)\right] p d X-\left[\frac{(1-\cos z)}{8}+\frac{1+\cos z}{8} G(v)\right] q d Y, \\
& M d x+E d t= \\
& \left\{\frac{(1-\cos w)}{8 c}+\frac{(1+\cos w)}{8 c} G(v)\right\} p d X+\left\{\frac{1-\cos z}{8 c}+\frac{1+\cos z}{8 c} G(v)\right\} q d Y .
\end{aligned}
$$




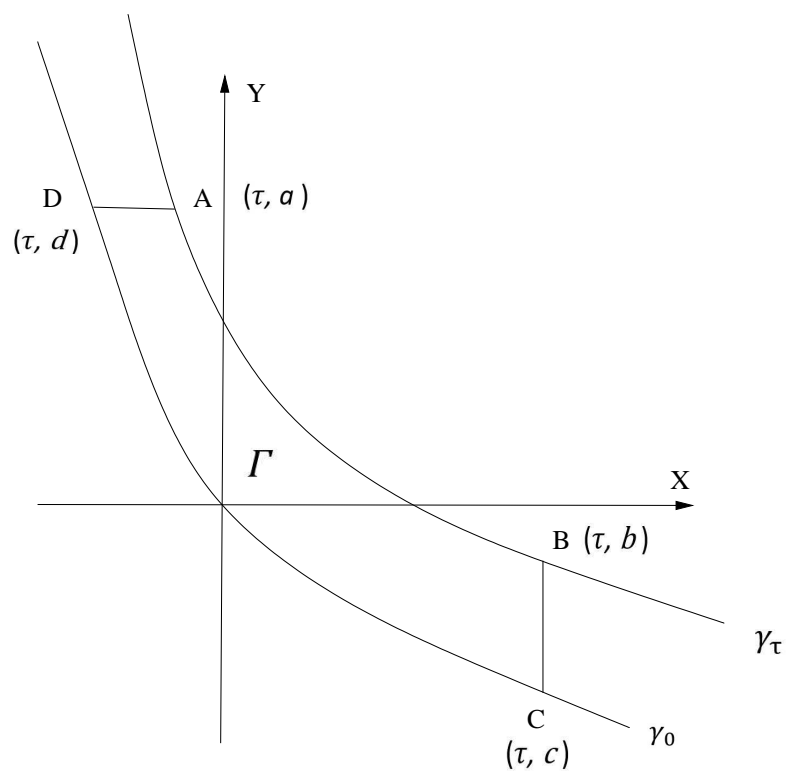

Figure 4 . The region $\Gamma$.

And we compute that

$$
\begin{aligned}
& \left\{\left[\frac{(1-\cos w)}{8}+\frac{(1+\cos w)}{8} G(v)\right] p\right\}_{Y} \\
= & \frac{-\sin w G(v) p}{8} \frac{c^{\prime}}{8 c^{2}}(\cos z-\cos w) q+\frac{(1+\cos w) g(v) p}{16} \frac{1}{4 c} \sin z q \\
& +\frac{1+\cos w}{8} G(v) \frac{c^{\prime}}{8 c^{2}}[\sin z-\sin w] p q \\
= & -\left\{\left[\frac{(1-\cos z)}{8}+\frac{1+\cos z}{8} G(v)\right] q\right\}_{X},
\end{aligned}
$$

and

$$
\begin{aligned}
& \left\{\left[\frac{(1-\cos w)}{8 c}+\frac{(1+\cos w)}{8 c} G(v)\right] p\right\}_{Y} \\
= & \frac{\sin w p}{64 c^{3}} c^{\prime}(\cos z-\cos w) q-\frac{\sin w p q}{64 c^{2}} g(v)(1+\cos z)(1+\cos w) \\
& +\frac{(1-\cos w) c^{\prime}}{64 c^{3}}[\sin z-\sin w] p q-\frac{(1-\cos w) p q}{64 c^{2}} \sin w g(v)(1+\cos z) \\
& -\frac{\sin w}{8 c} p G(v) \frac{c^{\prime}}{8 c^{2}}(\cos z-\cos w) q+\frac{(1+\cos w)}{8 c} G(v) \\
& \frac{c^{\prime}}{8 c^{2}}[\sin z-\sin w] p q+\frac{1+\cos w}{8 c} p g(v) \frac{1}{4 c} \sin z q \\
= & \left\{\left[\frac{1-\cos z}{8 c}+\frac{1+\cos z}{8 c} G(v)\right] q\right\}_{X} .
\end{aligned}
$$

Thus $\left\{E d x-\left(c^{2} M\right) d t\right\}, \quad\{M d x-E d t\}$ are closed.

To prove the inequality (1.9), We fixed some $\tau>0$, and the case $\tau<0$ is identical. We 
assume that for an arbitrary large $r>0$. We define the set

$$
\Gamma:=\{(X, Y): 0 \leq t(X, Y) \leq \tau, X \leq r, Y \leq r\} .
$$

We form the map $(X, Y) \mapsto(t, x)$ in the following pattern:

$$
A \mapsto(\tau, a), \quad B \mapsto(\tau, b), \quad C \mapsto(0, c), \quad D \mapsto(0, d),
$$

such that $a<b$ and $c>d$. Then, we can integrate the (5.4) along $\partial \Gamma$, the boundary of $\Gamma$.

$$
\begin{aligned}
& \int_{A B}\left\{\frac{(1-\cos w) p}{8}+\frac{(1+\cos w) p}{8} G(v)\right\} d X-\left\{\frac{(1-\cos z) q}{8}+\frac{(1+\cos z) q}{8} G(v)\right\} d Y \\
= & \int_{D C}\left\{\frac{(1-\cos w) p}{8}+\frac{(1+\cos w) p}{8} G(v)\right\} d X-\left\{\frac{(1-\cos z) q}{8}+\frac{(1+\cos z) q}{8} G(v)\right\} d Y \\
& -\int_{D A}\left\{\frac{(1-\cos w) p}{8}+\frac{(1+\cos w) p}{8} G(v)\right\} d X \\
& -\int_{C B}\left\{\frac{(1-\cos z) q}{8}+\frac{(1+\cos z) q}{8} G(v)\right\} d Y \\
\leq & \int_{D C}\left\{\frac{(1-\cos w) p}{8}+\frac{(1+\cos w) p}{8} G(v)\right\} d X-\left\{\frac{(1-\cos z) q}{8}+\frac{(1+\cos z) q}{8} G(v)\right\} d Y \\
\leq & \int_{d}^{c} \frac{1}{2}\left[v_{t}^{2}(0, x)+c^{2}(v(0, x)) v_{x}^{2}(0, x)+\frac{1}{2}(g(v(0, x))] d x .\right.
\end{aligned}
$$

Also,

$$
\begin{aligned}
& \int_{a}^{b} \frac{1}{2}\left[v_{t}^{2}(0, x)+c^{2}(v(0, x)) v_{x}^{2}(0, x)+\frac{1}{2} g(v(0, x))\right] d x \\
= & \int_{A B \cap\{\cos w \neq-1\}}\left\{\frac{(1-\cos w) p}{8}+\frac{(1+\cos w) p}{8} G(v)\right\} d X \\
& -\left\{\frac{(1-\cos z) q}{8}+\frac{(1+\cos z) q}{8} G(v)\right\} d Y \\
\leq & \mathcal{E}_{0} .
\end{aligned}
$$

Let $r \rightarrow \infty, a \rightarrow-\infty$ and $b \rightarrow+\infty$. We conclude that $\mathcal{E}(t) \leq \mathcal{E}_{0}$. Thus, the inequity (1.9) is proved.

Now, we prove the Lipschitz condition on the map $t \mapsto v(t, \cdot)$ in the $L^{2}$ distance. First, for any fixed time $\tau$, we define $\mu_{\tau}:=\mu_{\tau}^{-}+\mu_{\tau}^{+}$and $\mu_{\tau}$ is the positive measure on the real lines. We define $\mu_{\tau}^{-}, \mu_{\tau}^{+}$as follows.

we define $\Gamma_{\tau}:=\{(X, Y): t(X, Y) \leq \tau\}$ and let $\gamma_{\tau}$ be the boundary of $\Gamma_{\tau}$.

For any open interval $] a, b\left[\right.$, we define $A=\left(X_{A}, Y_{A}\right), B=\left(X_{B}, Y_{B}\right)$ be points on the $\gamma_{\tau}$ such that

$$
\begin{aligned}
& x(A)=a, \text { and } X_{P}-Y_{P} \leq X_{A}-Y_{A} \text { for all points } P \in \gamma_{\tau} \text { and } x(P) \leq a, \\
& x(B)=b, \text { and } X_{B}-Y_{B} \leq X_{P}-Y_{P} \text { for all points } P \in \gamma_{\tau} \text { and } x(P) \geq b .
\end{aligned}
$$

Then we have

$$
\mu_{\tau}:=\mu_{\tau}^{-}(] a, b[)+\mu_{\tau}^{+}(] a, b[),
$$



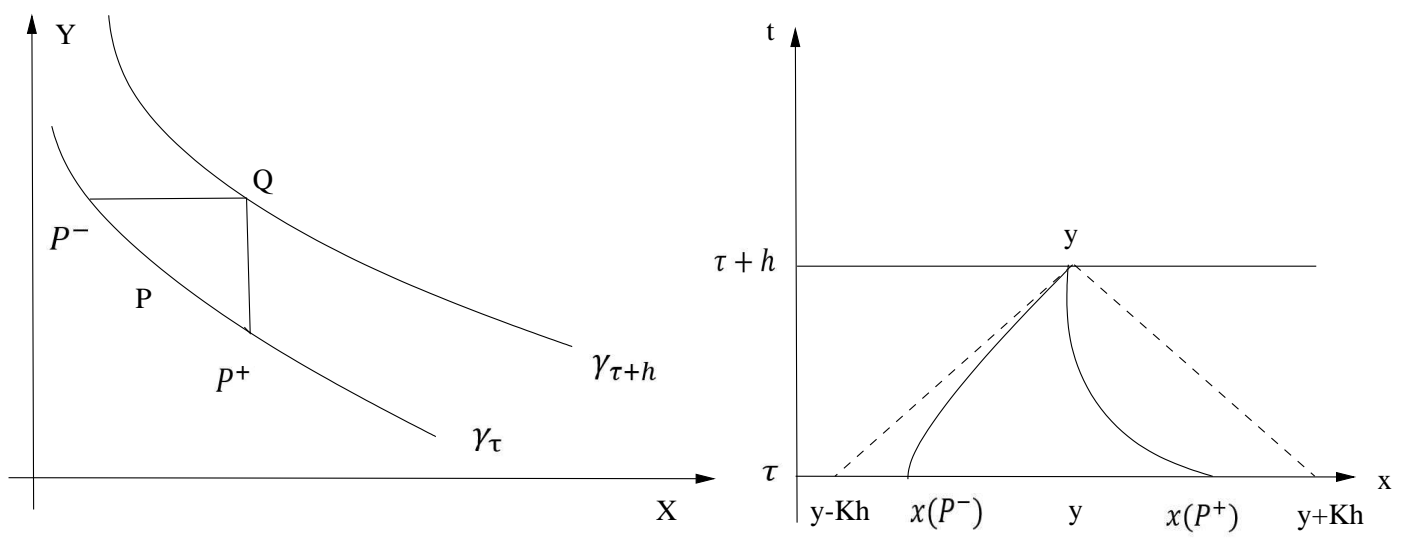

Figure 5. Proving Lipschitz condition.

and define that in the general case

$$
\begin{aligned}
& \mu_{\tau}^{-}(] a, b[):=\int_{A B}\left\{\frac{(1-\cos w) p}{8}+\frac{(1+\cos w) p}{8} G(v)\right\} d X, \\
& \mu_{\tau}^{+}(] a, b[):=\int_{A B}-\left\{\frac{(1-\cos z) q}{8}+\frac{(1+\cos z) q}{8} G(v)\right\} d Y .
\end{aligned}
$$

In the smooth case:

$$
\begin{aligned}
\mu_{\tau}^{-}(] a, b[) & :=\frac{1}{4} \int_{a}^{b} R^{2}(\tau, x) d x, \\
\mu_{\tau}^{+}(] a, b[) & :=\frac{1}{4} \int_{a}^{b} S^{2}(\tau, x) d x .
\end{aligned}
$$

Clearly, $\mu^{+}$and $\mu^{-}$are bounded positive measure. For all $\tau$, we have $\mu_{\tau}(\mathbb{R})=\mathcal{E}_{0}$ by (5.4). By (5.9)- (5.10) and (2.13) we compute that

$$
\begin{aligned}
\int_{a}^{b} c^{2} u_{x}^{2} d x=\int_{a}^{b} \frac{c^{2}(R-S)^{2}}{4 c^{2}} d x & =\int_{a}^{b} \frac{R^{2}-2 R S+S^{2}}{4} d x \\
& \leq \int_{a}^{b} \frac{R^{2}+S^{2}}{2} d x=2 \mu(] a, b[) .
\end{aligned}
$$

Thus, for arbitrary $a, b$ with $a<b$,

$$
|v(\tau, b)-v(\tau, a)|^{2} \leq|b-a| \int_{a}^{b} v_{x}(\tau, y) d y \leq|b-a| 2 \mathcal{K}^{2} \mu_{\tau}(] a, b[) .
$$

For given $y \in R$ and $h>0$, our goal is to estimate the $|v(\tau+h, y)-v(\tau, y)|$. We first denote that $\Gamma_{\tau+h}$ as the set $\Gamma_{\tau+h}:=\{(X, Y): t(X, Y) \leq \tau+h\}$ and denote that $\gamma_{\tau+h}$ to be the boundary of the set $\Gamma_{\tau+h}$.

Let $P=\left(P_{X}, P_{Y}\right)$ be points on $\gamma_{\tau+h}$ (as the figure $5($ a) shows) such that $x(P)=y$, and $X_{\tilde{P}}-Y_{\tilde{P}} \leq X_{P}-Y_{P}$ for all $\tilde{P} \in \gamma_{\tau}, x(\tilde{P}) \leq x(P)$.

Let $Q=\left(Q_{X}, Q_{Y}\right)$ be points on $\gamma_{\tau+h}$ such that $x(Q)=y$ and $X_{\tilde{Q}}-Y_{\tilde{Q}} \leq X_{Q}-Y_{Q}$ for all $\tilde{Q} \in \gamma_{\tau+h}, x(\tilde{Q}) \leq x(Q)$.

So $X_{P} \leq X_{Q}$ and $Y_{P} \leq Y_{Q}$. Let $P^{+}=\left(X_{Q}, Y^{+}\right) \in \gamma_{\tau}$ and $P^{-}=\left(X^{-}, Y_{Q}\right) \in \gamma_{\tau}$. 
As shown in the figure 5 , since the point $\left(\tau, x\left(P^{+}\right)\right)$lies on some characteristic curve with the speed $c(v) \leq \mathcal{K}$ and go through the point $(\tau+h, y)$, so $\left.x\left(P^{+}\right) \in\right] y, y+\mathcal{K} h[$. Also, $\left.x\left(P^{-}\right) \in\right] y-\mathcal{K} h, y[$, since point $(\tau, y)$ lies on some characteristic curve with the speed $-c(v) \geq-\mathcal{K}$ and go through the point $(\tau+h, y)$.

Thus, and by (2.30), we can compute that

$$
\begin{aligned}
\left|v(Q)-v\left(P^{+}\right)\right| & \leq \int_{Y^{+}}^{Y_{Q}}\left|v_{Y}\left(X_{Q}, Y\right)\right| d Y \\
& =\int_{Y^{+}}^{Y_{Q}}\left(\frac{1+\cos z}{4 c} q\right)^{\frac{1}{2}}\left(\frac{1-\cos z}{4 c} q\right)^{\frac{1}{2}} d Y \\
& \leq\left(\int_{Y^{+}}^{Y_{Q}} \frac{1+\cos z}{4 c} q d Y\right)^{\frac{1}{2}}\left(\int_{Y^{+}}^{Y_{Q}} \frac{1-\cos z}{4 c} q d Y\right)^{\frac{1}{2}} \\
& \leq\left(\int_{Y^{+}}^{Y_{Q}} \frac{1+\cos z}{4 c} q d Y+\frac{1+\cos w}{4 c} p d X\right)^{\frac{1}{2}}\left(\int_{Y^{+}}^{Y_{Q}} \frac{1-\cos z}{4 c} q d Y+\frac{1-\cos w}{4 c} p d Y\right)^{\frac{1}{2}} \\
& \leq\left(\int_{\tau}^{\tau+h} 1 d t\right)^{\frac{1}{2}}\left(\int_{P^{-}}^{P^{+}} \frac{1-\cos z}{4 c} q d Y+\frac{1-\cos w}{4 c} p d Y\right)^{\frac{1}{2}} \\
& \leq h^{\frac{1}{2}}\left(\int_{P^{-}}^{P^{+}} \frac{1-\cos z}{4 c} q d Y+\frac{1-\cos w}{4 c} p d Y\right)^{\frac{1}{2}} \cdot
\end{aligned}
$$

Thus

$$
\left|v(Q)-v\left(P^{+}\right)\right| \leq h^{\frac{1}{2}}\left(\int_{P^{-}}^{P^{+}} \frac{1-\cos z}{4 c} q d Y+\frac{1-\cos w}{4 c} p d Y\right)^{\frac{1}{2}} .
$$

So, by (5.11) and (5.12) we compute that

$$
\begin{aligned}
|v(\tau+h, x)-v(\tau, x)|^{2}= & \left|v(\tau+h, x)-v\left(t\left(P^{+}\right), x\left(P^{+}\right)\right)+v\left(t\left(P^{+}\right), x\left(P^{+}\right)\right)-v(\tau, x)\right|^{2} \\
\leq & 2\left\{v(\tau+h, x)-v\left(t\left(P^{+}\right), x\left(P^{+}\right)\right)\right\}^{2}+2\left\{v\left(t\left(P^{+}\right), x\left(P^{+}\right)\right)-v(\tau, x)\right\}^{2} \\
\leq & 2\left\{v(Q)-v\left(P^{+}\right)\right\}^{2}+2\left\{v\left(P^{+}\right)-v(P)\right\}^{2} \\
\leq & 2\left[h^{\frac{1}{2}}\left(\int_{P^{-}}^{P^{+}} \frac{1-\cos z}{4 c} q d Y+\frac{1-\cos w}{4 c} p d Y\right)^{\frac{1}{2}}\right]^{2} \\
& +2\left[2 \mathcal{K}^{2}(\mathcal{K} h) \mu_{\tau}(] x, x+h[)\right] \\
\leq & 4 h \mu_{\tau}(] x-\mathcal{K} h, x+\mathcal{K} h[)+4 \mathcal{K}^{3} h \mu_{\tau}(] x, x+h[) \\
\leq & 4 h \mu_{\tau}(] x-\mathcal{K} h, x+\mathcal{K} h[)\left(1+\mathcal{K}^{3}\right) .
\end{aligned}
$$

Thus, for all $h>0$,

$$
\|v(\tau+h, \cdot)-v(\tau, \cdot)\|_{L^{2}}=\left\{\int|v(\tau+h, x)-v(\tau, x)|^{2} d x\right\}^{\frac{1}{2}}
$$




$$
\begin{aligned}
& \leq\left\{\int 4\left(1+\mathcal{K}^{3}\right) h \mu_{\tau}(] x-\mathcal{K} h, x+\mathcal{K} h[)\right\}^{\frac{1}{2}} \\
& \leq\left\{4\left(\mathcal{K}^{3}+1\right) h^{2} \mu_{\tau}(\mathbb{R})\right\}^{\frac{1}{2}} \\
& \leq h\left[4\left(\mathcal{K}^{3}+1\right) \mathcal{E}_{0}\right]^{\frac{1}{2}} \\
& \leq|\tau+h-\tau| L, \\
\| v(\tau+h, \cdot) & -v(\tau, \cdot) \|_{L^{2}} \leq h\left[4\left(\mathcal{K}^{3}+1\right) \mathcal{E}_{0}\right]^{\frac{1}{2}},
\end{aligned}
$$

where $L=\left[4\left(\mathcal{K}^{3}+1\right) \mathcal{E}_{0}\right]^{\frac{1}{2}}$ is the Lipschitz constant. So, this proves the uniform Lipschitz continuous of the maps $t \mapsto v(t, \cdot)$.

\section{Regularity OF TRAJECTORIES}

In this section, we show that continuity of functions $t \mapsto v_{t}(t, \cdot)$ and $t \mapsto v_{x}(t, \cdot)$ as functions with function value in $L^{2}$. It completes the proof of Theorem 1.1.

We consider the that the initial data $\left(v_{0}\right)_{x}$ and $v_{1}$ are smooth functions with compact support. In this situation, the solution $v(X, Y)$ is smooth on the $X-Y$ plane. Fix some time $\tau$ and denote that $\Gamma_{\tau}:=\{(X, Y): t(X, Y) \leq \tau\} . \gamma_{\tau}$ is the boundary of set $\Gamma_{\tau}$. Then we claim that

$$
\left.\frac{d}{d t} v(t, \cdot)\right|_{t=\tau}=v_{t}(\tau, \cdot)
$$

By (2.21), (2.24), and (2.30),

$$
\begin{aligned}
v_{t}(\tau, x) & :=v_{X} X_{t}+v_{Y} Y_{t} \\
& =\frac{\sin w}{4 c} p \frac{2 c}{p(1+\cos w)}+\frac{\sin z}{4 c} q \frac{2 c}{q(1+\cos z)} \\
& =\frac{\sin w}{2(1+\cos w)}+\frac{\sin z}{2(1+\cos z)} .
\end{aligned}
$$

(6.2) define the value of $v_{t}(\tau, \cdot)$ at almost all the point of $x \in \mathbb{R}$. By the inequity (1.9) and $c(v) \geq \mathcal{K}^{-1}$,

$$
\int_{\mathbb{R}}\left|v_{t}(\tau, x)\right|^{2} d x \leq \mathcal{K}^{2} \mathcal{E}(\tau) \leq \mathcal{K}^{2} \mathcal{E}_{0}
$$

Next, to prove (6.1), given $\epsilon>0$, there exists finitely many disjoint intervals $\left[a_{i}, b_{i}\right]$ subsets of $\mathbb{R}$ with $i=1,2 \ldots N$. We call the $A_{i}, B_{i} \in \gamma_{\tau}$ with $x\left(A_{i}\right)=a_{i}, x\left(B_{i}\right)=b_{i}$. Then at every point $P$ in the $\operatorname{arcs} A_{i} B_{i}$ while $1+\cos (w(P))>\epsilon$ and $1+\cos (z(P))>\epsilon$,

$$
\min \{1+\cos (w(P)), 1+\cos (z(P))\} \leq 2 \epsilon .
$$

We call that $J:=\bigcup_{1 \leq i \leq N}\left[a_{i}, b_{i}\right]$ as the points $P$ along the curve $\gamma_{\tau}$ that does not contain in any of the $\operatorname{arcs} A_{i} B_{i}$ and denote that $J^{\prime}:=\mathbb{R} \backslash J$. Since $v(t, x)$ is smooth in a neighbourhood of the set $\{\tau\} \times J^{\prime}$ and by the differentiability of $v$ and apply the Minkowski's inequality,

$$
\lim _{h \rightarrow 0} \frac{1}{h}\left\{\int_{\mathbb{R}}\left|v(\tau+h, x)-v(\tau, x)-h v_{t}(\tau, x)\right|^{p} d x\right\}^{\frac{1}{p}}
$$




$$
\leq \lim _{h \rightarrow 0} \frac{1}{h}\left\{\int_{j}|v(\tau+h, x)-v(\tau, x)|^{p} d x\right\}^{\frac{1}{p}}+\left\{\int_{J}\left|v_{t}(\tau, x)\right|^{p} d x\right\}^{\frac{1}{p}} .
$$

Now, we estimate the measure of the bad set $J$. Since $(1+\cos w)<2 \epsilon(1-\cos w)$ and $(1+\cos z)<2 \epsilon(1-\cos z)$,

$$
\begin{aligned}
\operatorname{meas}(J) & =\int_{J} d x=\sum_{i} \int_{A_{i} B_{i}} \frac{(1+\cos w) p}{4} d X-\frac{(1+\cos z) q}{4} d Y \\
& \leq 2 \epsilon \sum_{i} \int_{A_{i} B_{i}} \frac{(1-\cos w) p}{4} d X-\frac{(1-\cos z) q}{4} d Y \\
& \leq 2 \epsilon \int_{\gamma_{\tau}} \frac{(1-\cos w) p}{4} d X-\frac{(1-\cos z) q}{4} d Y \\
& \leq 2 \epsilon \mathcal{E}_{0} .
\end{aligned}
$$

Using Hölder's inequality with exponents $\frac{2}{p}$ and $q$, we choose $q=\frac{2}{2-p}$ so that $\frac{p}{2}+\frac{1}{q}=1$. By (5.13),

$$
\begin{aligned}
\int_{J}|v(\tau+h, x)-v(\tau, x)|^{p} d x & \leq \operatorname{meas}(J)^{\frac{1}{q}}\left\{\int_{J}|v(\tau, x)-v(\tau, x)|^{2} d x\right\}^{\frac{p}{2}} \\
& \leq\left[2 \epsilon \mathcal{E}_{0}\right]^{\frac{1}{q}}+\left\{h\left[4\left(\mathcal{K}^{3}+1\right) \mathcal{E}_{0}\right]^{\frac{1}{2}}\right\}^{p}
\end{aligned}
$$

Thus,

$$
\begin{aligned}
& \lim _{h \rightarrow 0} \sup \frac{1}{h}\left\{\int_{J}|v(\tau+h, x)-v(\tau, x)|^{p} d x\right\}^{\frac{1}{p}} \\
\leq & {\left[2 \epsilon \mathcal{E}_{0}\right]^{\frac{1}{p q}}+h\left[4\left(\mathcal{K}^{3}+1\right) \mathcal{E}_{0}\right]^{\frac{1}{2}} . }
\end{aligned}
$$

Similarly, and by (6.3) we estimate that

Thus,

$$
\int_{J}\left|v_{t}(\tau, x)\right|^{p} d x \leq[\operatorname{meas}(J)]^{\frac{1}{q}}\left\{\int_{J}\left|v_{t}(\tau, x)\right|^{2} d x\right\}^{\frac{p}{2}} .
$$

$$
\left\{\int_{J}\left|v_{t}(\tau, x)\right|^{p} d x\right\}^{\frac{1}{p}} \leq\left[2 \epsilon \mathcal{E}_{0}\right]^{\frac{1}{p q}}\left[\mathcal{K}^{2} \mathcal{E}_{0}\right]^{\frac{1}{2}}
$$

Since $\epsilon>0$ is arbitrary, so we conclude that

$$
\lim _{h \rightarrow 0} \frac{1}{h}\left\{\int_{\mathbb{R}}\left|v(\tau+h, x)-v(\tau, x)-h v_{t}(\tau, x)\right|^{p} d x\right\}^{\frac{1}{p}}=0 .
$$

Next, we prove the continuity of the map $t \mapsto v_{t}$. First, we fix $\epsilon>0$ and consider disjoint intervals $\left[a_{i}, b_{i}\right]$ subsets of $\mathbb{R}$ with $i=1,2 \ldots N$. We call the $A_{i}, B_{i} \in \gamma_{\tau}$ with $x\left(A_{i}\right)=a_{i}$, $x\left(B_{i}\right)=b_{i}$. Since $v$ is a smooth function on the neighbourhood of $\{\tau\} \times J^{\prime}$. By Hölder's inequality and Minkowski's inequality, we estimate that

$$
\begin{aligned}
& \limsup _{h \rightarrow 0} \int\left|v_{t}(\tau+h, x)-v_{t}(\tau, x)\right|^{p} d x \\
& \leq \limsup \int_{h \rightarrow 0}\left|v_{t}(\tau+h, x)-v_{t}(\tau, x)\right|^{p} d x
\end{aligned}
$$




$$
\begin{aligned}
& \leq \limsup _{h \rightarrow 0}[\operatorname{meas}(J)]^{\frac{1}{q}}\left\{\int_{J}\left|v_{t}(\tau+h, x)-v_{t}(\tau, x)\right|^{2} d x\right\}^{\frac{q}{2}} \\
& \leq \lim \sup _{h \rightarrow 0}\left[2 \epsilon \mathcal{E}_{0}\right]^{\frac{1}{q}}\left\{\left\|v_{t}(\tau+h, \cdot)\right\|_{L^{2}}+\left\|v_{t}(\tau, \cdot)\right\|_{L^{2}}\right\}^{\frac{q}{2}} \\
& \leq\left[2 \epsilon \mathcal{E}_{0}\right]^{\frac{1}{q}}\left[4 \mathcal{E}_{0}\right]^{p} .
\end{aligned}
$$

Since the $\epsilon>0$ is arbitrary, so we prove the continuity.

For general initial data $\left(v_{0}\right)_{x}, v_{1} \in H^{1}$, we consider a sequence of initial data $v_{0}^{n} \rightarrow v_{0}$, $\left(v_{0}^{n}\right)_{x} \rightarrow\left(v_{0}\right)_{x}$, and $v_{1}^{n} \rightarrow v_{1}$ in $\in H^{1}$ for all $n \in \mathbb{N},\left(v_{0}^{n}\right)_{x}, v_{0}^{n}, v_{1}^{n} \in C_{c}^{\infty}$. The continuity of the map $t \mapsto v_{x}(t, \cdot)$ with values in $L^{p}$ and $1 \leq p<2$ can be proved in the same way as above.

\section{ENERGY CONSERVATION}

In this section, we provide proof of Theorem 1.3. First, we define the wave interaction potential as

$$
\Lambda(t):=\left(\mu_{t}^{-} \otimes \mu_{t}^{+}\right)\{(x, y): x>y\}
$$

where the $\mu_{t}^{-}$and $\mu_{t}^{+}$are defined in (5.7) and (5.8). And since $\mu_{t}^{-}$and $\mu_{t}^{+}$are absolutely continuous in Lebesgue measure, so (5.9) and (5.10) holds and (7.1) implies that

$$
\Lambda(t)=\frac{1}{4} \iint_{x>y} R^{2}(t, x) S^{2}(t, x) d x d y .
$$

Lemma 7.1. There exists a Lipschitz constant $L_{0}$ such that

$$
\Lambda(t)-\Lambda(s) \leq L_{0}(t-s),
$$

with $t>s>0$. So the map $t \mapsto \Lambda(t)$ has bounded variation.

The Lemma is proved later in this section.

To prove Theorem 1.3, we need to consider three sets

$$
\begin{aligned}
& \Omega_{1}:=\left\{(X, Y): w(X, Y)=-\pi, z(X, Y) \neq-\pi, c^{\prime}(v(X, Y)) \neq 0\right\}, \\
& \Omega_{2}:=\left\{(X, Y): w(X, Y) \neq \pi, z(X, Y)=-\pi, c^{\prime}(v(X, Y)) \neq 0\right\}, \\
& \Omega_{3}:=\left\{(X, Y): w(X, Y)=-\pi, z(X, Y)=-\pi, c^{\prime}(v(X, Y)) \neq 0\right\} .
\end{aligned}
$$

From (2.28), and since $w_{Y} \neq 0$ on $\Omega_{1}$ and $z_{X} \neq 0$ on $\Omega_{2}$, so that meas $\left(\Omega_{1}\right)=0$ and $\operatorname{meas}\left(\Omega_{2}\right)=0$.

We define $\Omega_{3}^{*}$ be the set of Lebesgue points of $\Omega_{3}$ and want to show that

$$
\operatorname{meas}\left(\left\{t(X, Y):(X, Y) \in \Omega_{3}^{*}\right\}\right)=0
$$

First, we fix point $P^{*} \in \Omega_{3}^{*}$ and $P^{*}:=\left(X^{*}, Y^{*}\right)$ and claim that for $h, k>0$,

$$
\lim _{h, k \rightarrow 0^{+}} \frac{\Lambda(\tau-h)-\Lambda(\tau+k)}{h+k}=+\infty .
$$

For arbitrary $\epsilon>0, \epsilon$ arbitrary small, we can find $\delta>0$ such that for any square $Q$ with length $l \leq \delta$ center at $P^{*}$, there exists a vertical segment $\sigma$ satisfying meas $\left(\Omega_{3} \cup \sigma\right) \geq(1-\epsilon) l$, 
and a horizontal segment $\sigma^{\prime}$ satisfying meas $\left(\Omega_{3} \cup \sigma^{\prime}\right) \geq(1-\epsilon) l$.

We define that

$$
\begin{aligned}
t^{+} & :=\max \left\{t(X, Y):(X, Y) \in \sigma \cup \sigma^{\prime}\right\}, \\
t^{-} & :=\min \left\{t(X, Y):(X, Y) \in \sigma \cup \sigma^{\prime}\right\} .
\end{aligned}
$$

By (4.4), for some constant $c_{0}>0$

$$
t^{+}-t^{-} \leq \int_{\sigma} \frac{(1+\cos w) p}{4 c} d X+\int_{\sigma^{\prime}} \frac{(1+\cos z) q}{4 c} d Y \leq c_{0}(\epsilon l)^{2} .
$$

(7.7) is Lipschitz continuous and vanished outside of a set of measure $\epsilon l$. Also, for some constant $c_{1}, c_{2}>0$,

$$
\Lambda\left(t^{-}\right)-\Lambda\left(t^{+}\right) \geq c_{1}(1-\epsilon)^{2} l^{2}-c_{2}\left(t^{+}+-t^{-}\right) .
$$

Since the choose of $\epsilon>0$ is arbitrary, so this implies (7.4). And by the Lemma 1, the map $t \mapsto \Lambda$ has bounded variation, so (7.4) implies (7.3).

Thus, the singular part of the $\mu_{t}$ is not trivial only if the set $\Omega_{4}:=\left\{P \in \gamma_{t}: w(P)=\right.$ $-\pi, z(P)=-\pi\}$ has positive one-dimensional measure. By the above analysis, this is restricted to a set where $c^{\prime} \neq 0$ and only happens for a set of time with measure zero.

\section{Proof of Lemma 1.}

From (2.11),

$$
\left\{\begin{array}{l}
\left(R^{2}\right)_{t}-\left(c R^{2}\right)_{x}=\frac{c^{\prime}}{2 c}\left(R^{2} S-S^{2} R\right)-R g(v), \\
\left(S^{2}\right)_{t}+\left(c S^{2}\right)_{x}=-\frac{c^{\prime}}{2 c}\left(R^{2} S-S^{2} R\right)-S g(v) .
\end{array}\right.
$$

We first provide an argument valid for $v=v(t, x)$ is smooth. (7.9) implies that

$$
\begin{aligned}
\frac{d}{d t}(4 \Lambda(t))= & \frac{d}{d t} \iint_{x>y} R^{2}(t, x) S^{2}(t, y) d x d y \\
= & \iint 2 R(t, x) S^{2}(t, y) c R_{x}(t, x)+2 S(t, y) R^{2}(t, x) c S_{x}(t, y) \\
& +2 S(t, y) R^{2}(t, x) \frac{c^{\prime}}{4 c}\left(S^{2}(t, x)-R^{2}(t, x)\right)+2 R(t, x) S^{2}(t, y) \frac{c^{\prime}}{4 c}\left(R^{2}(t, x)-S^{2}(t, x)\right) \\
& -R(t, x) S^{2}(t, y) g(v(t, x))-S(t, y) R^{2}(t, x) g(v(t, y)) d x d y \\
\leq & \iint c\left(S^{2} R^{2}\right)_{x}+\frac{c^{\prime}}{2 c}\left(R^{2}-S^{2}\right)\left(R S^{2}-S^{2} R\right) \\
& -R(t, x) S^{2}(t, y) g(v(t, x))-R^{2}(t, x) S(t, y) g(v(t, y)) d x d y \\
\leq & -2 \int c R^{2} S^{2} d x+\int\left(R^{2}+S^{2}\right) d x \cdot \int \frac{c^{\prime}}{2 c}\left|R^{2} S-S^{2} R\right| d x \\
& -\iint R(t, x) S^{2}(t, y) g(v(t, x))+R^{2}(t, x) S(t, y) g(v(t, y)) d x d y .
\end{aligned}
$$

And estimate the last term from the above calculation,

$$
\left|\iint R(t, x) S^{2}(t, y) g(v(t, x)) d x d y\right|
$$




$$
\begin{aligned}
& \leq \int S^{2}(t, y) d y \int|R(t, x) g(v(t, x))| d x \\
& \leq \mathcal{E}_{0}\|R(t, x)\|_{L^{2}}\|g(v(t, x))\|_{L^{2}} \\
& \leq \mathcal{E}_{0} \mathcal{E}_{0}^{\frac{1}{2}}\|v(t, x)\|_{L^{2}}\left\|\frac{g(v(t, x))}{v(t, x)}\right\|_{L^{\infty}} \\
& \leq \mathcal{E}_{0}^{2}
\end{aligned}
$$

Similarly,

$$
\begin{aligned}
& \left|\iint R^{2}(t, x) S(t, y) g(v(t, y)) d x d y\right| \\
& \leq \mathcal{E}_{0}^{2}
\end{aligned}
$$

Thus

$$
\frac{d}{d t}(4 \Lambda(t)) \leq-2 \mathcal{K}^{-1} \iint R^{2} S^{2} d x+4 \mathcal{E}_{0}\left\|\frac{c^{\prime}}{2 c}\right\|_{L^{\infty}} \int\left|R^{2} S-S^{2} R\right| d x+2 \mathcal{E}_{0}^{2},
$$

where $\mathcal{K}^{-1}$ is the lower bound for the speed $c(v)$. For each $\epsilon>0$, we have $|R| \leq \epsilon^{-\frac{1}{2}}+\epsilon^{\frac{1}{2}} R^{2}$. And pick any $\epsilon>0$ such that $\mathcal{K}^{-1}>4 \mathcal{E}_{0}\left\|\frac{c^{\prime}}{2 c}\right\|_{L^{\infty}} \cdot 2 \sqrt{\epsilon}$.

Thus

$$
\frac{d}{d t}(4 \Lambda(t)) \leq-\mathcal{K}^{-1} \int R^{2} S^{2} d x+\frac{16 \mathcal{E}_{0}^{2}}{\sqrt{\epsilon}}\left\|\frac{c^{\prime}}{2 c}\right\|_{L^{\infty}}+2 \mathcal{E}_{0}^{2} .
$$

This yields the $L^{1}$ estimate:

$$
\int_{0}^{\tau} \int\left(\left|R^{2} S\right|+\left|R S^{2}\right|\right) d x d t=\vartheta(1) \cdot\left[\Lambda(0)+\mathcal{E}_{0}^{2} \tau\right]=\vartheta(1) \cdot(1+\tau) \mathcal{E}_{0}^{2},
$$

Where $\vartheta(1)$ is defined as a quantity and its absolute value has a uniform bound depending only on $c(v)$. Also, the map $t \mapsto \Lambda(t)$ has bounded variation on any bounded interval. The smooth case is proved. The following provides a proof of Lemma 1 in general cases. For every $\epsilon>0$, there exists a constant $K_{\epsilon}$ satisfying that for all $w, z$,

$$
\begin{aligned}
& |\sin z(1-\cos w)-\sin w(1-\cos z)| \\
& \leq K_{\epsilon}\left[\tan ^{2}\left(\frac{w}{2}\right)+\tan ^{2}\left(\frac{z}{2}\right)\right](1+\cos w)(1+\cos z)+\epsilon(1-\cos w)(1-\cos z) .
\end{aligned}
$$

For fixed $0 \leq s<t$, consider the sets $\Gamma_{s}$ and $\Gamma_{t}$ as we defined in (5.5) and define $\Gamma_{s t}:=\Gamma_{t} \backslash \Gamma_{s}$. Recall that

We write that

$$
d x d t=\frac{p q}{8 c}(1+\cos w)(1+\cos z) d X d Y
$$

$$
\int_{s}^{t} \int_{-\infty}^{+\infty} \frac{1}{4}\left(R^{2}-S^{2}\right) d x d t=(t-s) \mathcal{E}_{0}
$$

$$
\int_{s}^{t} \int_{-\infty}^{+\infty} \frac{1}{4}\left(R^{2}-S^{2}\right) d x d t=\iint_{\Gamma_{s t}} \frac{p q}{32 c}(1+\cos w)(1+\cos z)\left[\tan ^{2}\left(\frac{z}{2}\right)+\tan ^{2}\left(\frac{w}{2}\right)\right] d X d Y .
$$


(7.13) holds only on the case that $v(t, x)$ is smooth while (7.14) holds for all cases. Combine (5.4), (5.7), (5.8) and apply (7.12)-(7.14), we obtain that

$$
\begin{aligned}
\Lambda(t)-\Lambda(s) \leq & \iint_{\Gamma_{s t}} \frac{(1-\cos w)(1-\cos z) p q}{64} d X d Y \\
& +\mathcal{E}_{0} \iint_{\Gamma_{s t}} \frac{c^{\prime} p q}{64 c^{2}}[\sin z(1-\cos w)-\sin w(1-\cos z)] d X d Y \\
& +\mathcal{E}_{0} \iint_{\Gamma_{s t}}\left\{-\frac{p q}{32 c^{2}} g(v)(1+\cos z) \sin w-\frac{\sin w G(v) p}{8} \frac{c^{\prime}}{8 c}(\cos z-\cos w) q\right. \\
& \left.+\frac{(1+\cos w) g(v) p}{32 c} \sin z q+\frac{(1+\cos w)}{32} \frac{c^{\prime}}{2 c^{2}} G(v)[\sin z-\sin w] p q\right\} d X d Y \\
\leq & \frac{1}{64} \iint_{\Gamma_{s t}}(1-\cos w)(1-\cos z) p q d X d Y \\
& +\mathcal{E}_{0} \iint_{\Gamma_{s t}} \frac{c^{\prime}}{64 c^{2}} p q\left\{K_{\epsilon}\left[\tan { }^{2}\left(\frac{w}{2}\right)+\tan { }^{2}\left(\frac{z}{2}\right)\right](1+\cos w)(1+\cos z)\right. \\
& +\epsilon(1-\cos w)(1-\cos z)\} d X d Y+\mathcal{E}_{0} \iint_{\Gamma_{s t}}\left\{-\frac{p q}{32 c^{2}} g(v)(1+\cos z) \sin w\right. \\
& -\frac{\sin w G(v) p}{8} \frac{c^{\prime}}{8 c}(\cos z-\cos w) q+\frac{(1+\cos w) g(v) p}{32 c} \sin z q \\
& \left.+\frac{(1+\cos w)}{32} \frac{c^{\prime}}{2 c^{2}} G(v)[\sin z-\sin w] p q\right\} d X d Y \\
\leq & K(t-s),
\end{aligned}
$$

for a suitable constant $K$. Thus, Lemma 1 is proved.

Acknowledgement: I would like to thank Qingtian Zhang for suggesting this project and helpful discussion. I am grateful for the encouragement and support I was given throughout my thesis. Also, thanks to the Mathematics Department at UC Davis for this magnificent opportunity.

\section{REFERENCES}

[1] A. Bressan, and G. Chen, Generic regularity of conservative solutions to a nonlinear wave equation, Ann. I. H. Poincare-AN, 34 (2017), 335-354.

[2] A. Bressan, and G. Chen, Lipschitz metrics for a class of nonlinear wave equations, Arch. Rat. Mech. Anal., 226 (2017), 1303-1343.

[3] A. Bressan, G. Chen, and Q. T. Zhang, Unique conservative solutions to a variational wave equation, Arch. Rat. Mech. Anal., 217 (2015), 1069-1101.

[4] A. Bressan, and T. Huang, Representation of dissipative solutions to a nonlinear variational wave equation, Comm. Math. Sci., 14 (2016), 31-53.

[5] A. Bressan, and Y. X. Zheng, Conservative solutions to a nonlinear variational wave equation, Comm. Math. Phys. 266 (2006), no. 2, 471-497.

[6] G. Chen, and Y. X. Zheng, Singularity and existence for a wave system of nematic liquid crystals, $J$. Math. Anal. Appl., 398 (2013), 170-188. 
[7] R. T. Glassey, J. K. Hunter, and Y. X. Zheng, Singularities and oscillations in a nonlinear variational wave equation, The IMA Volumes in Mathematics and its applications, 91 (1997), 37-60.

[8] H. Holden, and X. Raynaud, Global semigroup of conservative solutions of the nonlinear variational wave equation, Arch. Ration. Mech. Anal., 201 (2011), 871-964.

[9] J. C. Huang, and Y. X. Zheng, No blow-up to a variational wave equation in liquid crystals, J. Math. Phys. 57 (2016), no. 2, 021506, 10 pp.

[10] J. K. Hunter, and R. A. Saxton, Dynamics of director fields, SIAM J. Appl. Math., 51(1991), 1498-1521.

[11] F. Lin, Nonlinear theory of defects in nematics liquid crystals: phase transitation and flow phenomena, Commun. Pure Appl. Math. 42 (1989), 789-814.

[12] D. K. Yang, Effects of Electric Field on Liquid Crystals. Fundamentals of liquid crystal devices. John Wiley \& Sons, 2014, 107-112.

[13] P. Zhang, and Y. X. Zheng, Conservative solutions to a system of variational wave equations of nematic liquid crystals, Arch. Rat. Mech. Anal., 195 (2010), 701-727.

[14] P. Zhang, and Y. X. Zheng, Energy conservative solutions to a one-dimensional full variational wave system, Comm. Pure Appl. Math., 65 (2012), 683-726.

[15] P. Zhang, and Y. X. Zheng, Existence and uniqueness of solutions of an asymptotic equation arising from a variational wave equation with general data, Arch. Rat. Mech. Anal., 155 (2000), 49-83.

[16] P. Zhang, and Y. X. Zheng, On the global weak solutions to a variational wave equation, Handbook of Differential Equations: Evolutionary Equations. Vol. 2. North-Holland, 2005, 561-648.

[17] P. Zhang, and Y. X. Zheng, On the second-order asymptotic equation of a variational wave equation, Proc. Roy. Soc. Edinburgh, 132 (2002), 483-509.

[18] P. Zhang, and Y. X. Zheng, Rarefactive solutions to a nonlinear variational wave equation of liquid crystals, Comm. Partial Differential Equations, 26 (2001), no. 3-4, 381-419.

[19] P. Zhang, and Y. X. Zheng, Singular and rarefactive solutions to a nonlinear variational wave equation, Chinese Ann. Math. Series B, 22 (2001), 159-170.

[20] P. Zhang, and Y. X. Zheng, Weak solutions to a nonlinear variational wave equation, Arch. Rat. Mech. Anal., 166 (2003), 303-319.

[21] P. Zhang, and Y. X. Zheng, Weak solutions to a nonlinear variational wave equation with general data, Ann. I. H. Poincare-An, 22 (2005), 207-226. 